\title{
High Performance Reduced Order Modeling techniques based on optimal energy quadrature. Application to geometrically non-linear multiscale inelastic material modeling
}

\author{
Manuel Caicedo ${ }^{\mathrm{a}}$, Javier L. Mroginski ${ }^{\mathrm{b}}$, Sebastian Toro ${ }^{\mathrm{c}}$, Marcelo Raschi $^{\mathrm{a}}$, Alfredo Huespe ${ }^{\mathrm{c}, \mathrm{d}}$, Javier \\ Oliver ${ }^{\mathrm{a}, \mathrm{d}}$ \\ ${ }^{a}$ CIMNE - Centre Internacional de Metodes Numerics en Enginyeria, Campus Nord UPC, Mdul C-1, c/ Jordi Girona \\ 1-3, 08034, Barcelona, Spain \\ ${ }^{b}$ CONICET, Argentine Council for Science and Technology, Argentina. \\ ${ }^{c}$ CIMEC-UNL-CONICET, Predio Conicet, Ruta Nac. $168 \mathrm{~s} / n$ - Paraje El Pozo, 3000 Santa Fe Argentina. \\ ${ }^{d}$ E.T.S d'Enginyers de Camins, Canals i Ports, Technical University of Catalonia (BarcelonaTech), Campus Nord \\ UPC, Mòdul C-1, c/ Jordi Girona 1-3, 08034, Barcelona, Spain
}

\begin{abstract}
A High-Performance Reduced-Order Model (HPROM) technique, previously presented by the authors in the context of hierarchical multiscale models for non linear-materials undergoing infinitesimal strains, is generalized to deal with large deformation elasto-plastic problems.

The proposed HPROM technique uses a Proper Orthogonal Decomposition (POD) procedure to build a reduced basis of the primary kinematical variable of the micro-scale problem, defined in terms of the micro-deformation gradient fluctuations. Then a Galerkin-projection, onto this reduced basis, is utilized to reduce the dimensionality of the micro-force balance equation, the stress homogenization equation and the effective macro-constitutive tangent tensor equation. Finally, a reduced goal-oriented quadrature rule is introduced to compute the non-affine terms of these equations.

Main importance in this paper is given to the numerical assessment of the developed HPROM technique. The numerical experiments are performed on a micro-cell simulating a randomly distributed set of elastic inclusions embedded into an elasto-plastic matrix. This micro-structure is representative of a typical ductile metallic alloy. The HPROM technique applied to this type of problem displays high computational speed-ups, increasing with the complexity of the finite element model.

From these results, we conclude that the proposed HPROM technique is an effective computational tool for modeling, with very large speed-ups and acceptable accuracy levels with respect to the high-fidelity case, the multiscale behavior of heterogeneous materials subjected to large deformations involving two well-separated scales of length.
\end{abstract}

Keywords: High-Performance Reduced Order Modeling (HPROM), Multiscale Modeling, Computational Homogenization, Reduced Order Quadrature (ROQ) 


\section{Symbols related to the HPROM technique utilized in the paper}

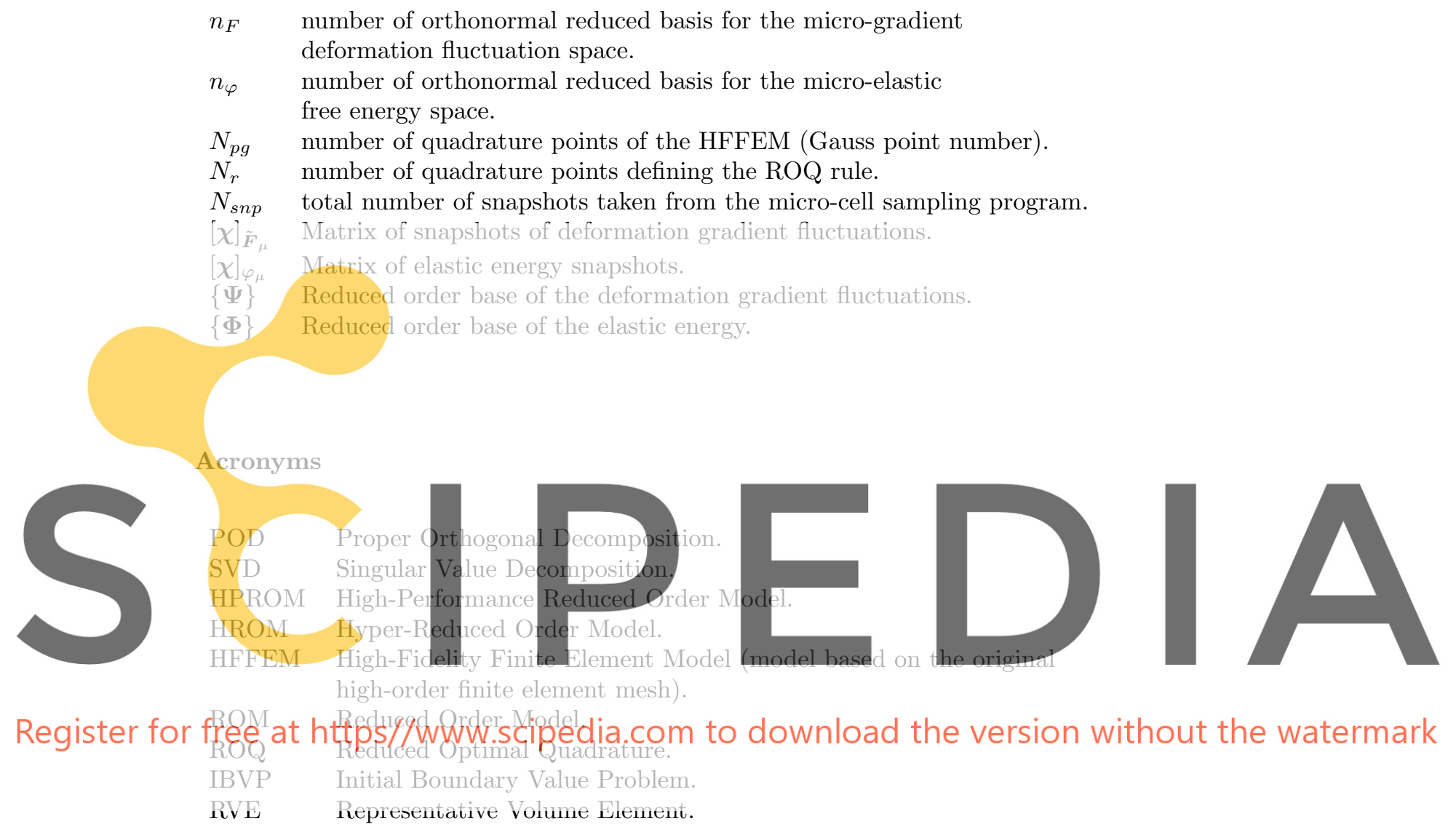

\section{Introduction}

Several reasons can be invoked in favor of adopting a two-scale hierarchical computational material homogenization technique, such as shown in [1] and [2], as a tool of analysis. This conceptual framework, frequently called $\mathrm{FE}^{2}$ approach by some authors ([3]), is becoming a widely utilized tool in the Computational Mechanics community, mainly to evaluate the effective properties of heterogeneous materials ([4]).

However, this technique still has some issues which limits its application to industrial problems. In fact, one of the main characteristic features of this approach remarked in the present context, refers to the required high computational cost to process the information computed at the micro-scale level. This information is obtained after solving a huge number of almost identical micro-mechanical problems, but notably, that information is then filtered out and only a small quantity of data is up-scaled at the macro-scale problem. An even more remarkable feature is that the space of data, driving these huge 
number of micro-mechanical problems, can be parametrized in a space of only four dimensions for plane problems or six dimensions for 3D problems.

From this perspective, it seems reasonable to wonder if a model reduction technique, providing approximated solutions, could give a favorable trade-off between computational cost and fidelity loss of results. We think that the answer to this question is a clear "yes", and furthermore, we also think that techniques based on dimensionality reduction and hyper-reduction of the small scale problem become a good option to get this target, such as explored by [5] and [6].

Following this goal, the purpose of this work is to generalize a version of the reduced-order model based on the Proper Orthogonal Decomposition (POD) and a reduced goal-oriented integration rule, previously presented by the authors in [7], aiming at a different range of applications. In the present work, we extend the HPROM technique for modeling non-linear geometrical behavior in multi-scale modeling via computational homogenization. Typically, large deformation problems subjected to small rotation regimes, observed in multi-scale homogenization problems arising in a wide range of material modeling applications.

The so-developed model is then assessed by determining the effective properties of a rather standard metallic composite which characterization, by means of a phenomenological mono-scale material model, has shown to be difficult
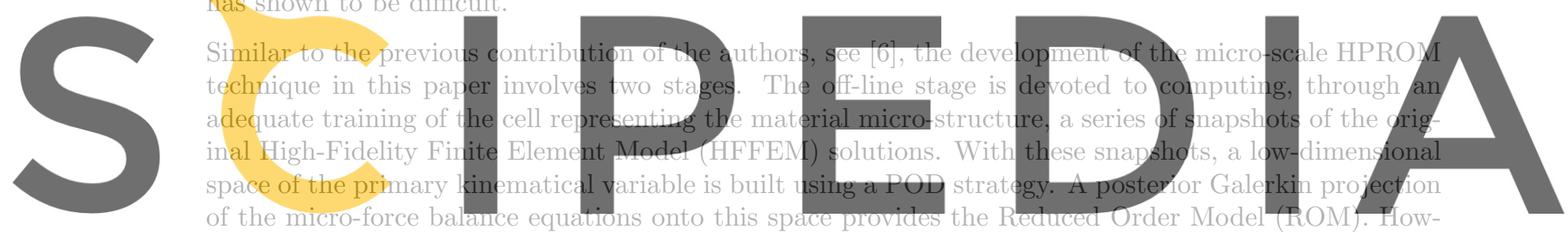

ever, as noted elsewhere (see [5], [8]), the integral of the non-linear micro-force balance equation terms,

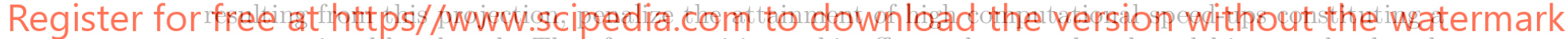

computational bottleneck. Therefore, to mitigate this effect, a hyper-reduced model is next developed; the name "hyper-reduced is taken from [5]. In the present paper, the hyper-reduction is attained by introducing a "Reduced Optimal Quadrature (ROQ) rule, which integrates accurately the POD modes of the elastic internal energy obtained from the solution snapshots above mentioned. The ROQ rule has been taken from [7] and preserves the Lagrangian structure of the mechanical problem, see [9].

The considered homogenization procedure is only valid for simulating the stable regime of the homogenized material. Such as commented in [10] and [11], in order to preserve the consistency of the problem mathematical formulation after losing the macro-stability, a characteristic length has to be extracted from the micro-cell model and used to regularize the homogenized model at the macroscale. Since this paper is focused on studying the performance of the proposed HPROM technique in the geometrically nonlinear regime, this issue is not addressed here, and therefore, the HPROM numerical assessments are restricted to the range where the macro-scale remain stable.

The paper is organized as follows. Section 2 presents a brief description of the multi-scale technique addressed to simulate heterogeneous materials. This topic is the object of study in this paper. In particular, sub-Section 2.4 describes several micro-cell Initial Boundary Value Problem (IBVP) formulations which are posteriorly utilized for implementing the ROM and ROQ procedures.

Section 3 summarizes the foundation and development of the HPROM technique, constituted by the 
ROM and ROQ procedures, for computing the micro-cell solutions. The ROM procedure is briefly presented in sub-Section 3.1, while the ROQ procedure in sub-Section 3.2. Section 4 describes the sampling program for testing the micro-cell HFFEM.

The numerical assessment of the HPROM technique is addressed in Section 5. We study the truncation and sampling errors associated with the micro-cell training program. Also, through a full multiescale test we evaluate the attained computation time speed-ups versus the errors introduced by the HPROM technique. The comparison of these results with that obtained using a HFFEM strategy gives the adequate support in favor of the HPROM technique. In the final part of the paper, we present the conclusions of this study.

\section{A Two-scale Model Under Finite Strain Regimes}

The computational multiscale modeling of heterogeneous material computes the stress-strain relationship by solving an IBVP formulated at the length scale at which the material micro-structure can be identified. Then, two length scales naturally arise in the problem: the macro-scale, of similar order to the structural size $h$, and the micro-scale, of similar order to the material heterogeneity size $h_{\mu}$, see Figure 1 . Both length scales are assumed to be well-separated.
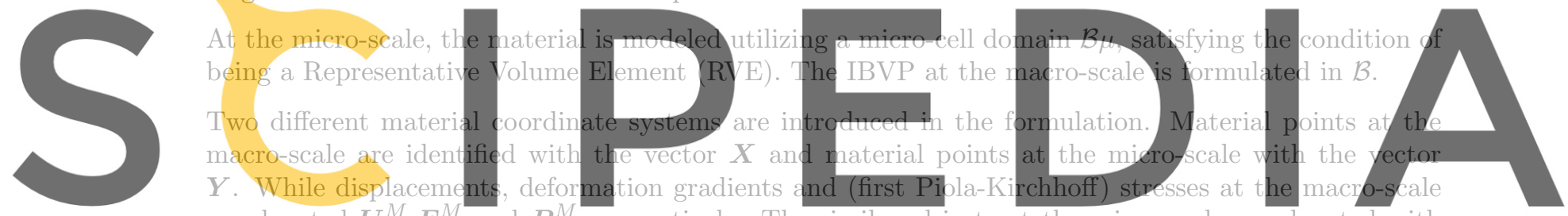

are denoted $U^{M}, F^{M}$ and $P^{M}$, respectively. The similar objects at the micro-scale are denoted with

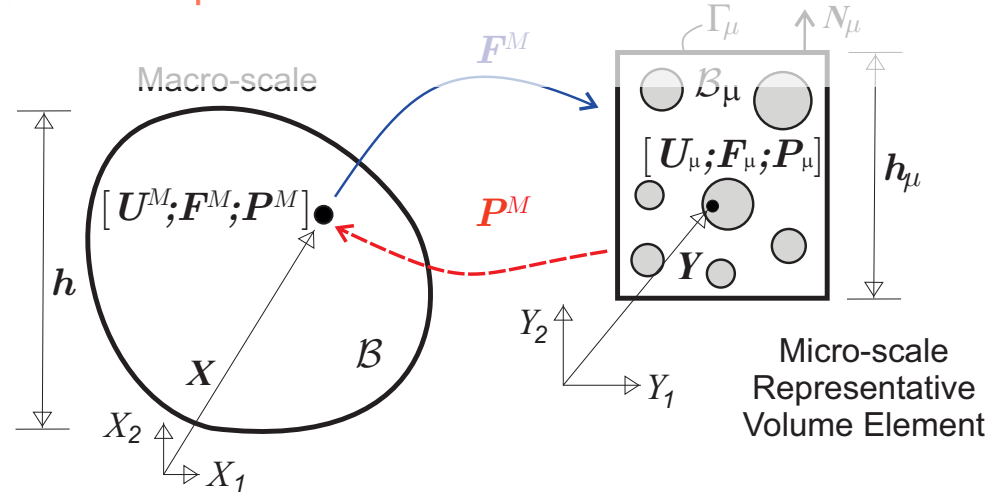

Figure 1: Two-scale model scheme and notation utilized to identify objects at each scale.

\subsection{Admissible Kinematics at the RVE}

The two-scale model formulation in this Section follows a similar description of that presented in [12] and [13]. Let us consider the micro-scale domain $\mathcal{B}_{\mu}$, sketched in Fig. 1, of size $h_{\mu}$ and boundary $\Gamma_{\mu}$, 
with unit normal vector denoted by $\boldsymbol{N}_{\mu}$. The microscopic displacement field, denoted in the material description $\boldsymbol{U}_{\mu}$, can be expressed as the addition of three terms:

$$
\boldsymbol{U}_{\mu}(\boldsymbol{Y}, t ; \boldsymbol{X})=\boldsymbol{U}^{M}(\boldsymbol{X}, t)+\left[\boldsymbol{F}^{M}(\boldsymbol{X}, t)-\boldsymbol{I}\right] \boldsymbol{Y}+\tilde{\boldsymbol{U}}_{\mu}(\boldsymbol{Y}, t)
$$

where $\boldsymbol{U}^{M}$ is the macro-scale displacement, the second term is a displacement field which gradient is $\nabla_{\boldsymbol{Y}}(\bullet)=\boldsymbol{F}^{M}(\boldsymbol{X}, t)-\boldsymbol{I}=\nabla_{\boldsymbol{X}} \boldsymbol{U}^{M}(\boldsymbol{X}, t)$ and $\tilde{\boldsymbol{U}}_{\mu}$ is a micro-displacement field fluctuation which becomes the primary unknown variable in the governing equations for the RVE conventional IBVP formulated as PROBLEM I (summarized in next sub-Sections). According to Eq. (1), the micro-deformation gradient can be expressed as the addition of two terms:

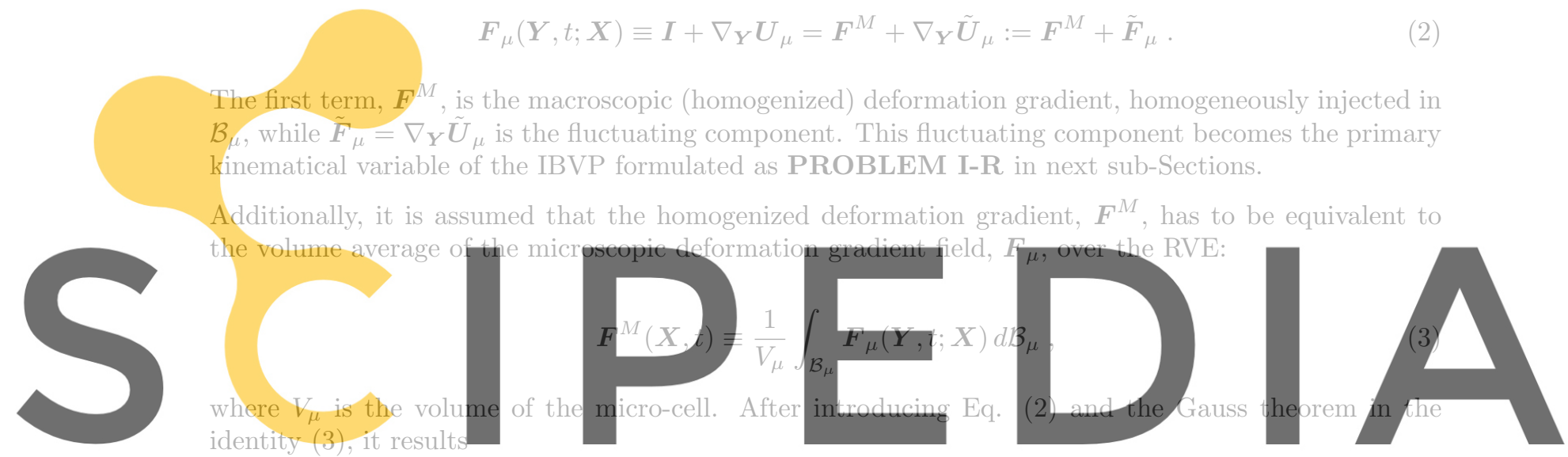

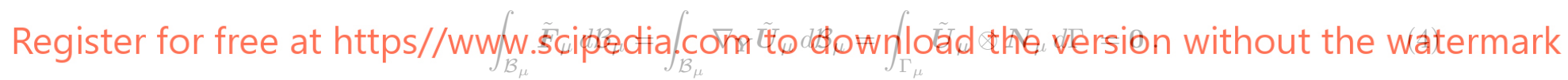

This equation induces a natural constraint on the possible RVE fluctuation fields of displacements and deformation gradients. Then, fields $\tilde{\boldsymbol{U}}_{\mu}$ and $\tilde{\boldsymbol{F}}_{\mu}$ only satisfying Eq. (4) are considered as kinematically admissible fields. Therefore, the minimally constrained set of kinematically admissible microdisplacements fluctuations $\mathcal{U}_{\mu}^{U}$ is defined as:

$$
\mathcal{U}_{\mu}^{U} \equiv\left\{\tilde{\boldsymbol{U}}_{\mu} \mid \int_{\mathcal{B}_{\mu}} \nabla_{\boldsymbol{Y}} \tilde{\boldsymbol{U}}_{\mu} d \mathcal{B}_{\mu}=\int_{\Gamma_{\mu}} \tilde{\boldsymbol{U}}_{\mu} \otimes \boldsymbol{n} d \Gamma=\mathbf{0}\right\}
$$

\subsection{Hill-Mandel Principle: Micro-scale Equilibrium Problem and Stress Homogenization}

The scale bridging equations for stresses can be derived by assuming the variational format of the commonly used Hill-Mandel Principle which establishes that the macroscopic virtual stress power $\boldsymbol{P}^{M}$ : $\delta \boldsymbol{F}^{M}$ must be equal to the volume average of the micro-virtual stress power $\boldsymbol{P}_{\mu}: \delta \boldsymbol{F}_{\mu}$ when the virtual kinematical terms, $\delta \boldsymbol{F}^{M}$ and $\delta \boldsymbol{F}_{\mu}$, are connected through Eq. (2) and Eq. (4). Here, and in the 
following, the symbol $\delta(\bullet)$ means the difference between two arbitrary elements of the corresponding functional space. This variational principle is mathematically expressed as:

$$
\begin{aligned}
\boldsymbol{P}^{M}: \delta \boldsymbol{F}^{M}= & \frac{1}{V_{\mu}} \int_{\mathcal{B}_{\mu}} \boldsymbol{P}_{\mu}:\left(\delta \boldsymbol{F}^{M}+\nabla_{\boldsymbol{Y}} \delta \tilde{\boldsymbol{U}}_{\mu}\right) d \mathcal{B}_{\mu}, \\
& \forall \delta \tilde{\boldsymbol{U}}_{\mu} \in \mathcal{V}_{\mu}^{U}, \quad \forall \delta \boldsymbol{F}^{M} \in \mathbb{R}^{n_{d i m} \times n_{d i m}} .
\end{aligned}
$$

The admissible space of micro-displacement fluctuations $\mathcal{V}_{\mu}^{U}$ is identical to $\mathcal{U}_{\mu}^{U}\left(\mathcal{V}_{\mu}^{U} \equiv \mathcal{U}_{\mu}^{U}\right)$, while the space of admissible macro-deformation gradient is the full space of second order tensors denoted $\mathbb{R}^{n_{\text {dim }} \times n_{\text {dim }}}\left(n_{\text {dim }}\right.$ is the space dimension).

As a consequence of Eq. (6), the following micro-scale equilibrium equation is obtained:

$$
\int_{\mathcal{B}_{\mu}} \boldsymbol{P}_{\mu}: \nabla_{\boldsymbol{Y}} \delta \tilde{\boldsymbol{U}}_{\mu} d \mathcal{B}_{\mu}=0 \quad \forall \delta \tilde{\boldsymbol{U}}_{\mu} \in \mathcal{V}_{\mu}^{U}
$$

as well as the stress homogenization equation:
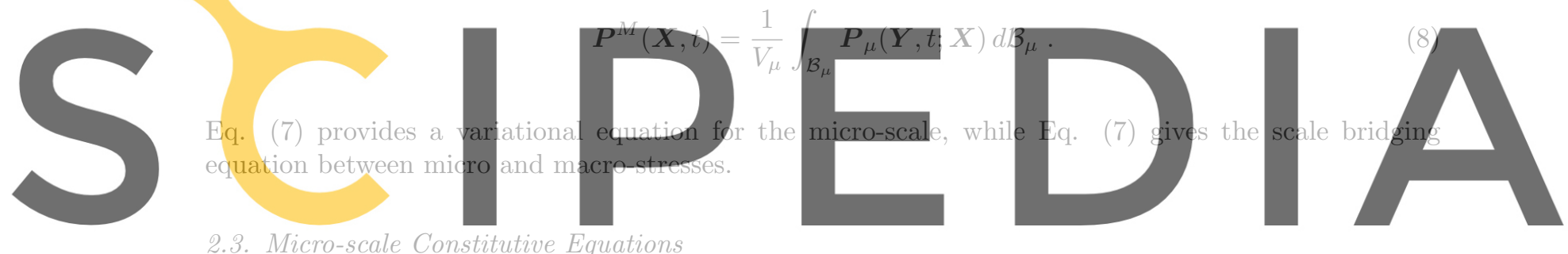

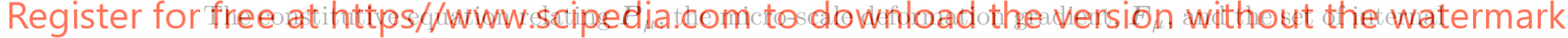 \\ variables, $\alpha_{\mu}$, at the micro-scale is here generically written as the stress-strain relationship}

$$
\boldsymbol{P}_{\mu}=\hat{\boldsymbol{P}}_{\mu}\left(\overline{\boldsymbol{F}}_{\mu}, \alpha_{\mu}\right)
$$

supplemented with the evolution equation of the internal variables

$$
\dot{\alpha}_{\mu}=g\left(\boldsymbol{F}_{\mu}, \dot{\boldsymbol{F}}_{\mu}, \alpha_{\mu}\right) .
$$

In particular, when the micro-components of the heterogeneous material follow an elasto-plastic constitutive law, the conventional multiplicative decomposition of the micro-deformation gradient $\boldsymbol{F}_{\mu}=\boldsymbol{F}_{\mu}^{e} \boldsymbol{F}_{\mu}^{p}$ is assumed, where $\boldsymbol{F}_{\mu}^{e}$ and $\boldsymbol{F}_{\mu}^{p}$ are the elastic and plastic deformation gradients, respectively. Also, an additive decomposition of the micro-free energy, $\varphi_{\mu}$, into elastic, $\varphi_{\mu}^{e}$, and plastic, $\varphi_{\mu}^{p}$, energy terms

$$
\varphi_{\mu}\left(\boldsymbol{F}_{\mu}, \boldsymbol{F}_{\mu}^{p}, \alpha_{\mu}\right)=\varphi_{\mu}^{e}\left(\boldsymbol{F}_{\mu}, \boldsymbol{F}_{\mu}^{p}\right)+\varphi_{\mu}^{p}\left(\alpha_{\mu}\right),
$$

is assumed.

In this case, the first Piola-Kirchhoff stress in the reference configuration, see [14], is obtained as

$$
\hat{\boldsymbol{P}}_{\mu}=\frac{\partial \varphi_{\mu}^{e}}{\partial \boldsymbol{F}_{\mu}} .
$$


Also, by considering the tangent constitutive tensor at the micro-scale $\mathbb{A}_{\mu}=\partial \hat{\boldsymbol{P}}_{\mu} / \partial \boldsymbol{F}_{\mu}$, the tensor of effective moduli $\mathbb{A}$ is computed as

$$
\mathbb{A}(\boldsymbol{X}, t)=\frac{\partial \boldsymbol{P}^{M}}{\partial \boldsymbol{F}^{M}}=\frac{1}{V_{\mu}} \int_{\mathcal{B}_{\mu}} \mathbb{A}_{\mu}(\boldsymbol{Y}, t)\left(\mathbb{I}+\mathbb{L}_{\mu}(\boldsymbol{X}, \boldsymbol{Y}, t)\right) d \mathcal{B}_{\mu},
$$

where $\mathbb{I}$ is the fourth order identity tensor and $\mathbb{L}_{\mu}=\partial\left(\nabla_{\boldsymbol{Y}} \tilde{\boldsymbol{U}}_{\mu}\right) / \partial \boldsymbol{F}^{M}$ is the localization tensor that can be obtained using Eq. (7). A complete description of the resulting homogenized macroscopic model can be found in [12].

\subsection{Micro-scale Initial Boundary Value Problem Formulations}

Three alternative and equivalent micro-scale IBVP formulations are described in this Section. The first one is a conventional formulation, widely utilized in the computational homogenization community resulting from the set of equations (5), (7) and the evolution equations for the internal variables of the component constitutive equations, at the micro-scale.

The second formulation is introduced with the objective of reducing the dimension of the space representing the field $F_{\mu}$.

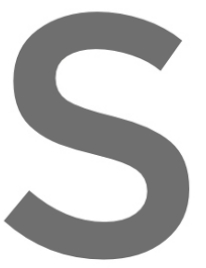

The third formulation is

2.4.1. Conventional

The set of Eqs. (5)-(t)
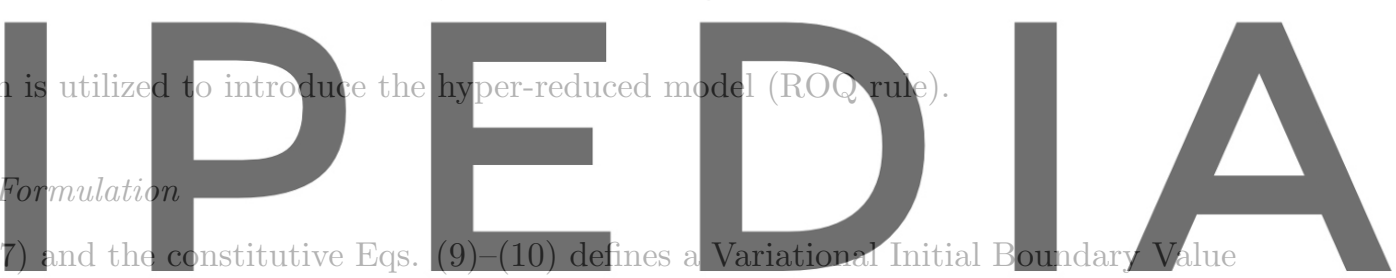

Problem (VIBVP) at the RVE. This problem is identified as PROBLEM I and is summarized in the

Register for free at https//www.scipedia.com to download the version without the watermark

PROBLEM I: (RVE variational displacement fluctuation-based problem)

Given the macro-scale deformation gradient, $\boldsymbol{F}$, the space of displacement fluctuations, $\mathcal{U}_{\mu}^{U}$, and the space of virtual kinematically admissible displacement fluctuations $\mathcal{V}_{\mu}^{U}$, with:

$$
\mathcal{U}_{\mu}^{U}=\mathcal{V}_{\mu}^{U}:=\left\{\tilde{\boldsymbol{U}}_{\mu} \mid \int_{\mathcal{B}_{\mu}} \nabla_{\boldsymbol{Y}} \tilde{\boldsymbol{U}}_{\mu} d \mathcal{B}_{\mu}=\int_{\Gamma_{\mu}} \tilde{\boldsymbol{U}}_{\mu} \otimes \boldsymbol{N}_{\mu} d \Gamma=\mathbf{0}\right\}
$$

find $\tilde{\boldsymbol{U}}_{\mu} \in \mathcal{U}_{\mu}^{U}$ such that:

$$
\begin{gathered}
\int_{\mathcal{B}_{\mu}} \hat{\boldsymbol{P}}_{\mu}\left(\boldsymbol{F}_{\mu}, \alpha_{\mu}\right): \nabla_{\boldsymbol{Y}} \delta \tilde{\boldsymbol{U}}_{\mu} d \mathcal{B}_{\mu}=0 ; \quad \forall \delta \tilde{\boldsymbol{U}}_{\mu} \in \mathcal{V}_{\mu}^{U} ; \\
\dot{\alpha}_{\mu}=g\left(\boldsymbol{F}_{\mu}, \dot{\boldsymbol{F}}_{\mu}, \alpha_{\mu}\right)
\end{gathered}
$$




\subsubsection{Formulation in Deformation Gradient Fluctuations}

As it will be shown later, it is convenient to rephrase PROBLEM I taking the micro-deformation gradient fluctuation, $\tilde{\boldsymbol{F}}_{\mu}$, as a primal variable instead of $\tilde{\boldsymbol{U}}_{\mu}$. Let us consider the space $\mathbb{F}_{\mu}$ of microdeformation gradient functions that are kinematically compatible. This space is characterized by (see $[15])$ :

$$
\mathbb{F}_{\mu}:=\left\{\tilde{\boldsymbol{F}}_{\mu} \in \mathbb{R}^{n_{\text {dim }} \times n_{\text {dim }}} \mid \nabla \wedge \tilde{\boldsymbol{F}}_{\mu}=\mathbf{0}\right\}
$$

Notice that the compatibility equation in (17) 1 is linear and homogeneous, which is a crucial fact to reduce the dimension of the space approaching the deformation gradient fluctuation field, $\tilde{F}_{\mu}$, through a POD technique.

\section{Taking into account Eq. (2) and constraint (4), PROBLEM I can be rephrased as:}

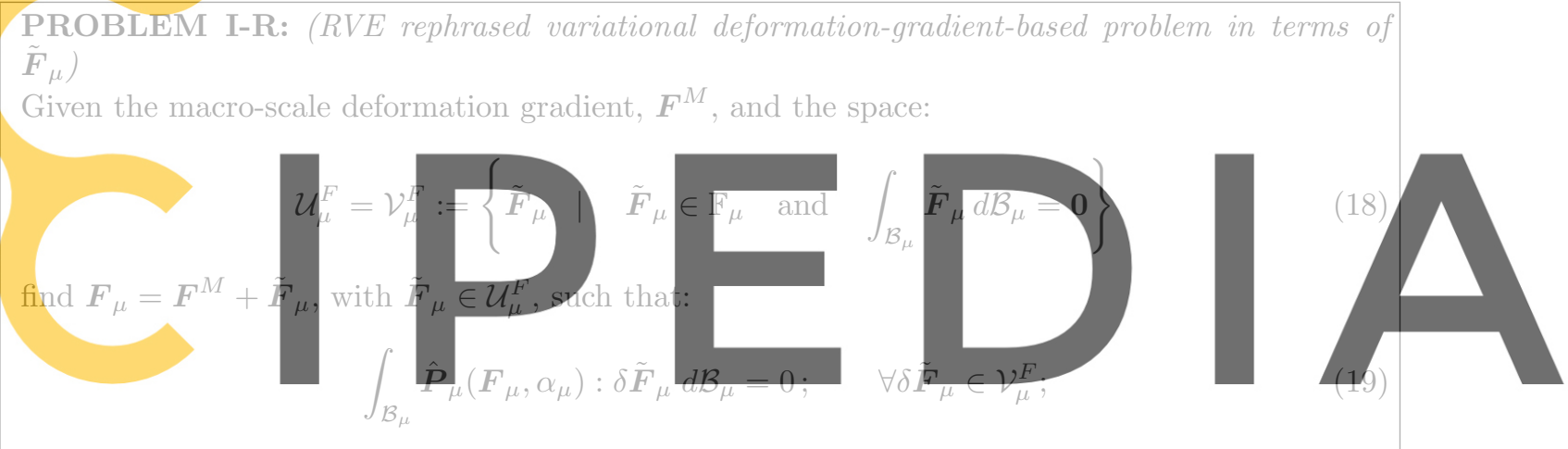

\section{Register for free at https//www.scipedia.cam to down hopd the version without the 2 watermark}

It can be proved that PROBLEM I-R is equivalent to PROBLEM I, where, in the later, the dependent variables (micro-scale First Piola-Kirchhoff stress $\boldsymbol{P}_{\mu}$ ) are now given in terms of the microdeformation gradient fluctuations, $\tilde{\boldsymbol{F}}_{\mu}$. The displacement fluctuations field, $\tilde{\boldsymbol{U}}_{\mu}$, could be recovered through a displacement-recovery procedure based on an additional integration at the reference configuration of the equation $\tilde{\boldsymbol{F}}_{\mu}=\nabla_{\boldsymbol{Y}} \tilde{\boldsymbol{U}}_{\mu}$. A consistent variational procedure for reaching this objective is provided in the reference [7].

\subsubsection{Formulation Based on the Elastic Free Energy}

After considering the stress-strain relationship, Eq. (12), the self-equilibrium of the micro-stresses can be imposed as a stationary point of the potential elastic energy with frozen $\boldsymbol{F}^{M}$ and internal variables (i.e. the overall elastic energy over the micro-cell is stationary with arbitrary variations of $\tilde{\boldsymbol{F}}_{\mu}{ }^{2}$ ), as

\footnotetext{
${ }^{1}$ The indicial notation of the compatibility equation is: $(\nabla \wedge \boldsymbol{\zeta})_{q l}=\epsilon_{l i p} \frac{\partial \boldsymbol{\zeta}_{p q}}{\partial \boldsymbol{X}_{i}}$, where $\epsilon$ is the permutation tensor.

${ }^{2}$ Stationarity is strictly considered only for infinistesimal variations of $\tilde{\boldsymbol{F}}_{\mu}$.
} 
follows:

$$
\begin{aligned}
\delta_{\boldsymbol{F}_{\mu}}\left(\int_{\mathcal{B}_{\mu}} \varphi_{\mu}^{e} d \mathcal{B}_{\mu}\right)_{\left[\boldsymbol{F}^{M}, \alpha_{\mu}\right]} & =\int_{\mathcal{B}_{\mu}}\left(\frac{\partial \varphi_{\mu}^{e}}{\partial \boldsymbol{F}_{\mu}}: \delta \boldsymbol{F}_{\mu}\right)_{\left[\boldsymbol{F}^{M}, \alpha_{\mu}\right]} d \mathcal{B}_{\mu}= \\
& =\int_{\mathcal{B}_{\mu}} \boldsymbol{P}_{\mu}: \delta \tilde{\boldsymbol{F}}_{\mu} d \mathcal{B}_{\mu}=\mathbf{0} \quad ; \quad \forall \delta \tilde{\boldsymbol{F}}_{\mu} \in \mathcal{U}_{\mu}^{F}
\end{aligned}
$$

where, the last identity is obtained after using Eq. (12) and Eq. (2) to obtain the admissible variation $\delta \boldsymbol{F}_{\mu}=\delta \tilde{\boldsymbol{F}}_{\mu}$. Subindex $\left[\boldsymbol{F}^{M}, \alpha_{\mu}\right]$ in Eq. (21) means that variables $\boldsymbol{F}^{M}$ and $\alpha_{\mu}$ are hold fixed when imposing variations of $\tilde{\boldsymbol{F}}_{\mu}$.

The equilibrium equation (21) has to be considered jointly with the evolution equation (10) to describe the complete IBVP. Then, the micro-scale mechanical problem can be re-formulated as shown in the following Box.

PROBLEM II: (RVE variational problem based on the elastic potential stationarity)

Given the macro-scale deformation gradient, $\boldsymbol{F}$, and the space $\mathcal{U}_{\mu}^{F}$, find $\boldsymbol{F}_{\mu}=\boldsymbol{F}+\tilde{\boldsymbol{F}}_{\mu}$, with $\tilde{F}_{\mu} \in \mathcal{U}_{\mu}^{F}$, such that:
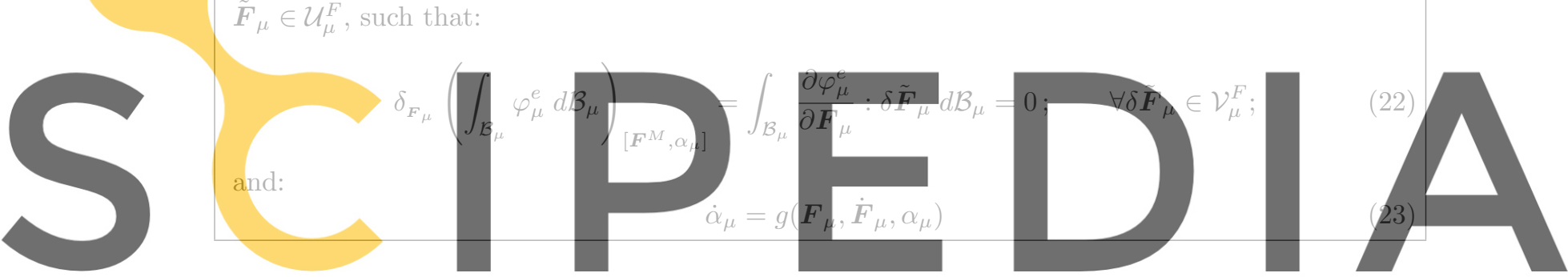

3. Hyper Reduced-Order Model of the Micro-scale Problem

Register for free at https//www.scipedia.com to download the version without the watermark

The proposed hyper-reduction strategy is based on the following sequential steps: initially, as described in sub-Section 3.1, a set of reduced basis for spanning two micro-state variables, the deformation gradient fluctuations and the elastic free energy, of the micro-cell problem are obtained through a POD procedure.

Then, the Reduced Order Model (ROM) is built by projecting the problem PROBLEM I-R onto the low-dimensional space of deformation gradient fluctuations. This step is described in next sub-Section 3.1.1.

In a subsequent step, a Reduced-Order Quadrature (ROQ) rule is introduced. With this rule, a low computational cost procedure for evaluating the volume integral in PROBLEM II , the homogenized stress Eq. (8) and the effective constitutive tangent tensor Eq. (13), is attained. This step is described in next sub-Section 3.2.

\subsection{Projection of State Variables in Low-dimensional Spaces}

To reduce the dimensionality of the kinematical variable $\tilde{\boldsymbol{F}}_{\mu}$, the original micro-cell HFFEM is used to sample the corresponding solution space through several training trajectories. A number of solutions, from these trajectories, are collected. Each sampled trajectory corresponds to a specific loading/unloading macro-strain history $\boldsymbol{F}^{M}(s)$ injected onto the microscale model. Here, the parameter $s \in\left[0, t_{\max }\right]$ represents the RVE loading history until reaching the pseudo-time $t_{\text {max }}$. 
Let us consider a given i-th solution gathered from a training trajectories. We identify the i-th column vector $\chi_{\tilde{\boldsymbol{F}}_{\mu}}^{i}$ (with $[\boldsymbol{\chi}]_{\tilde{\boldsymbol{F}}_{\mu}} \in \mathbb{R}^{4 N_{p g}}$ ) as the collection and piling-up of the deformation gradient fluctuations $\tilde{\boldsymbol{F}}_{\mu}$, of all the mesh quadrature points. Considering that the number of conventional quadrature Gauss points in the HFFEM are $N_{p g}$ and the space dimension is $n_{d i m}=2$ (i.e. $2 \mathrm{D}$ problems), then, the vector $\chi_{\tilde{\boldsymbol{F}}_{\mu}}^{i}$ is given by

$$
\chi_{\tilde{F}_{\mu}}^{i}=\left[\begin{array}{c}
\vdots \\
\left(\tilde{F}_{\mu}^{i}\right)_{11}^{k} \\
\left(\tilde{F}_{\mu}^{i} k_{22}^{k}\right. \\
\left(\tilde{F}_{\mu}^{i}\right)_{12}^{k} \\
\left(\tilde{F}_{\mu}^{i}\right)_{21}^{k} \\
\vdots
\end{array}\right] \in \mathbb{R}^{4 N_{p g}} \quad ; \quad k=\left[1, \ldots, N_{p g}\right] .
$$

where supraindex $k$ indicates the value at the corresponding $k$-th Gauss point. This vector represents a snapshot of the HFFEM solutions.

The snapshot matrix of deformation gradient fluctuations is built with the collection of the the $N_{s n p}$ snapshot vectors:
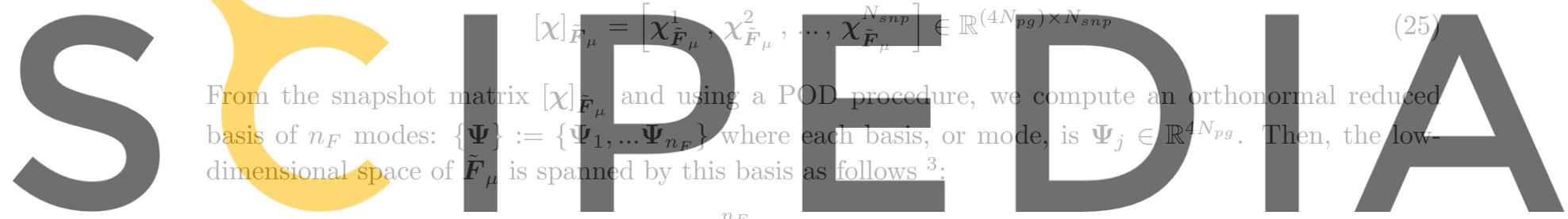

$$
\tilde{F}_{\mu}(\boldsymbol{Y}, t)=\sum^{n_{F}} \Psi_{j}(\boldsymbol{Y}) c_{j}(t)=\Psi(\boldsymbol{Y}) \mathrm{c}(t),
$$

Register for free at https//www.scipedia.coim to download the version without the watermark

where the coefficients $c$ are the time-dependent parameters of the linear combination. In the last identity, we have simplified the notation by introducing the matrix $\Psi \in \mathbb{R}^{\left(4 N_{p g}\right) \times n_{F}}$ and the vector $\mathrm{c} \in \mathbb{R}^{n_{F}}$, collecting the set of basis vectors $\left\{\boldsymbol{\Psi}_{j}\right\}$ and parameters $c_{j}$, respectively.

In the same way, and following a similar approach, snapshots of the elastic free energy, for all trajectories, are also gathered in the vector

$$
\chi_{\varphi_{\mu}^{e}}^{i}=\left[\begin{array}{c}
\vdots \\
\left(\left(\varphi_{\mu}^{e}\right)^{i}\right)^{k} \\
\vdots
\end{array}\right] \in \mathbb{R}^{N_{p g}} \quad ; \quad k=\left[1, \ldots, N_{p g}\right]
$$

and collected in the matrix: $[\chi]_{\varphi_{\mu}^{e}} \in \mathbb{R}^{N_{p g} \times N_{s n p}}$ :

$$
[\chi]_{\varphi_{\mu}^{e}}=\left[\chi_{\varphi_{\mu}^{e}}^{1}, \chi_{\varphi_{\mu}^{e}}^{2}, \ldots, \chi_{\varphi_{\mu}^{e}}^{N_{s n p}}\right] \in \mathbb{R}^{N_{p g} \times N_{s n p}}
$$

\footnotetext{
${ }^{3}$ Here, we introduce an abuse of notation. The expression $\boldsymbol{\Psi}_{j}(\boldsymbol{Y}) \in \mathbb{R}^{4}$ should be interpreted as the one-to-one mapping from the vector $\boldsymbol{\Psi}_{j} \in \mathbb{R}^{4 N_{p g}}$ to $N_{p g}$ vectors $\boldsymbol{\Psi}_{j}(\boldsymbol{Y}) \in \mathbb{R}^{4}$ where $\boldsymbol{Y}$ takes the $N_{p g}$ discrete values of the Gauss point positions. This mapping is a redistribution of the components of $\boldsymbol{\Psi}_{j}$.
} 
Then, using a POD technique, a reduced orthonormal basis, $\{\boldsymbol{\Phi}\}$, of $n_{\varphi}$ modes can be computed and the elastic free energy field is spanned using this low-dimensional basis:

$$
\varphi_{\mu}^{e}\left(\boldsymbol{F}_{\mu}^{e}(\boldsymbol{Y}, t), t\right)=\sum_{j=1}^{n_{\varphi}} \boldsymbol{\Phi}_{j}(\boldsymbol{Y}) f_{j}(t)=\boldsymbol{\Phi}(\boldsymbol{Y}) \boldsymbol{f}(t),
$$

where the vector $\boldsymbol{f}$ collects the time-dependent parameters of the elastic free energy linear combination. In the last equation, we have also simplified the notation by introducing the matrix $\boldsymbol{\Phi} \in \mathbb{R}^{N_{p g} \times n_{\varphi}}$.

The success of constructing properly a POD bases for $\tilde{\boldsymbol{F}}_{\mu}$ and $\varphi_{\mu}^{e}$ relies on gathering appropriate snapshots from the loading/unloading history representing the full space of solutions of the micro-cell HFFEM.

\subsubsection{Reduced Order Model (ROM)}

Utilizing the same projection defined by Eq. (26) to approach elements of the functional spaces $\mathcal{U}_{\mu}^{F}$ and $\mathcal{V}_{\mu}^{F}$, the BVP PROBLEM I-R can be re-formulated using a low-dimensional approach. In this case, Eq. (19) is written as

$$
\int_{\mathcal{B}_{\mu}} \hat{\boldsymbol{P}}_{\mu}\left(\boldsymbol{F}^{\mathbf{M}}+\boldsymbol{\Psi} \mathbf{c}, \alpha_{\mu}\right): \mathbf{\Psi}(\boldsymbol{Y}) \delta \mathbf{c} d \mathcal{B}_{\mu}=0 ; \quad \forall \delta \mathbf{c} \in \mathbb{R}^{n_{F}}
$$

We recall that all the basis vectors in $\{\boldsymbol{\Psi}\}$ satisfy the condition of belonging to $\mathcal{V}_{\mu}^{F}$. Therefore, any function spanned by $\boldsymbol{\Psi}$ also satisfies the same constraint ${ }^{4}$.

Eq. (30) jointly with the evolution equations of the internal variables (10) define the ROM variational problem.

\subsection{High-Performance Reduced Order Model (HPROM)}

The following the ideas are taken from the so-called cubature methods, described in [16] and [8], we propose a Reduced Optimal Quadrature (ROQ) rule to integrate the non-linear term arising in PROBLEM II .

The technique uses the low-dimensional expansion of the elastic free energy given by Eq. (29). Then, a reduced quadrature rule, similar to that proposed in [16], is introduced satisfying the following condition imposed on every mode $\boldsymbol{\Phi}_{j}$ :

$$
\begin{aligned}
\int_{\mathcal{B}_{\mu}} \boldsymbol{\Phi}_{j}(\boldsymbol{Y}) d \mathcal{B}_{\mu} & \approx \int_{\mathcal{B}_{\mu}}^{r e d} \boldsymbol{\Phi}_{j}(\boldsymbol{Y}) d \mathcal{B}_{\mu}= \\
& =\sum_{k=1}^{N_{r}} \boldsymbol{\Phi}_{j}\left(\boldsymbol{z}_{k}\right) \omega_{k} \quad ; \quad \text { for: } j=1, \ldots, n_{\varphi}
\end{aligned}
$$

\footnotetext{
${ }^{4}$ Since Eq. (17) is homogeneous, linear combinations of basis fulfilling strain compatibility give rise to compatible strains.
} 
where $\boldsymbol{z}_{k}$ are $N_{r}$ specific quadrature points in $\mathcal{B}_{\mu}$. Particularly, these points $\boldsymbol{z}_{k}$ are selected among the Gauss integration points corresponding to the original high-order finite element mesh. The terms $\omega_{k}$ are weights satisfying

$$
\sum_{k=1}^{N_{r}} \omega_{k}=V_{\mu} \quad \text { and } \quad \omega_{k} \geq 0\left(\text { for } k=1, \cdots, N_{r}\right) .
$$

Remark 1: a key issue to develop a feasible a ROQ scheme is to define an efficient and high performance algorithm to select the appropriate quadrature points, among the original global set of Gauss points, and to compute their corresponding weights. The algorithm we use in this work to attain this objective has been taken from [16].

Using the reduced quadrature, we compute the integral of the elastic free energy as

$$
\int_{\mathcal{B}_{\mu}} \varphi_{\mu}^{e}\left(\boldsymbol{F}_{\mu}, \boldsymbol{F}_{\mu}^{p}\right) d \mathcal{B}_{\mu} \approx \int_{\mathcal{B}_{\mu}}^{r e d} \varphi_{\mu}^{e}\left(\boldsymbol{F}_{\mu}, \boldsymbol{F}_{\mu}^{p}\right) d \mathcal{B}_{\mu}=\sum_{j=1}^{n_{\varphi}}\left(\sum_{k=1}^{N_{r}} \boldsymbol{\Phi}_{j}\left(\boldsymbol{z}_{k}\right) \omega_{k}\left(\boldsymbol{z}_{k}\right)\right) \boldsymbol{f}_{j} .
$$

Since the integration domain $\mathcal{B}_{\mu}$ is the same on both integral in Eq. (33), and they differ only on the used quadrature rule, $\int_{\mathcal{B}_{\mu}}(\cdot) d \mathcal{B}_{\mu}$ and $\int_{\mathcal{B}_{\mu}}^{\text {red }}(\cdot) d \mathcal{B}_{\mu}$, we assume that differentiation with respect to the argument $\boldsymbol{F}_{\mu}$ of the kernel in both expressions, keep the approximation consistent, i.e.

$$
\boldsymbol{P}^{M}=\frac{1}{V_{\mu}} \int_{\mathcal{B}_{\mu}} \frac{\partial \varphi_{\mu}^{e}\left(\boldsymbol{F}_{\mu}, \boldsymbol{F}_{\mu}^{p}\right)}{\partial \boldsymbol{F}^{M}} d \mathcal{B}_{\mu} \approx \frac{1}{V_{\mu}} \int_{\mathcal{B}_{\mu}}^{r e d} \frac{\partial \varphi_{\mu}^{e}\left(\boldsymbol{F}_{\mu}, \boldsymbol{F}_{\mu}^{p}\right)}{\partial \boldsymbol{F}^{M}} d \mathcal{B}_{\mu}
$$

where the first-Piola-Kirchhoff expression, Eq. (12), has been replaced. Using a similar argument, the equilibrium equation in PROBLEM II can be rewritten using the reduced quadrature as follows

$$
\begin{aligned}
& \delta_{\boldsymbol{F}_{\mu}}\left(\int_{\mathcal{B}_{\mu}} \varphi_{\mu}^{e} d \mathcal{B}_{\mu}\right)_{\left[\boldsymbol{F}^{M}, \alpha_{\mu}\right]} \approx \delta_{\boldsymbol{F}_{\mu}}\left(\int_{\mathcal{B}_{\mu}}^{r e d} \varphi_{\mu}^{e} d \mathcal{B}_{\mu}\right)_{\left[\boldsymbol{F}^{M}, \alpha_{\mu}\right]}= \\
& \int_{\mathcal{B}_{\mu}}^{r e d}\left(\frac{\partial \varphi_{\mu}^{e}}{\partial \boldsymbol{F}_{\mu}}: \delta \boldsymbol{F}_{\mu}\right)_{\left[\boldsymbol{F}^{M}, \alpha_{\mu}\right]} d \mathcal{B}_{\mu}=\int_{\mathcal{B}_{\mu}}^{r e d} \boldsymbol{P}_{\mu}: \delta \boldsymbol{F}_{\mu} d \mathcal{B}_{\mu}=0
\end{aligned}
$$

Additionally, the consistent derivation of Eq. (34) demands that the effective moduli, in Eq. (13), has to be rewritten using the same reduced quadrature

$$
\mathbb{A}(\boldsymbol{X}, t) \approx \frac{1}{V_{\mu}} \int_{\mathcal{B}_{\mu}}^{r e d} \mathbb{A}_{\mu}\left(\mathbb{I}+\mathbb{L}_{\mu}\right) d \mathcal{B}_{\mu} .
$$

Therefore, the integral terms in Eqs. (34), (35) and (36) can be computed using the rule:

$$
\int_{\mathcal{B}_{\mu}}^{\text {red }}(\bullet)(\boldsymbol{Y}) d \mathcal{B}_{\mu}=\sum_{k=1}^{N_{r}}(\bullet)\left(\boldsymbol{z}_{k}\right) \omega_{k}
$$

Remark 2: the accuracy obtained using the ROQ scheme in Eqs. (34), (35) and (36) will be, implicitly, proven through the consistency analyzes in Section 5. 


\section{RVE Configuration and Sampling Program Description}

\subsection{RVE off-line Sampling Program}

The driving force which activates the micro-cell mechanical problem is the macro-scale deformation gradient $\boldsymbol{F}^{M}$ that is injected from the macro-scale onto the micro-cell domain. It means that the components of $\boldsymbol{F}^{M}$ can be taken as parameters to define the micro-cell sampling program. Particularly for 2D problems, $\boldsymbol{F}^{M}$ has four independent components.

In accordance with this fact, we design the micro-cell sampling program by defining a network of points in the $\mathbb{R}^{4}$ space. The coordinates of each point identify the four parameters, $\Delta F_{11}, \Delta F_{22}, \Delta F_{12}$ and $\Delta F_{21}$. Then, every RVE training trajectory is associated with one network point by defining the RVE time-loading process through the deformation gradient:

$$
\boldsymbol{F}^{M}(s)=\boldsymbol{I}+s\left[\begin{array}{ll}
\Delta F_{11} & \Delta F_{12} \\
\Delta F_{21} & \Delta F_{22}
\end{array}\right] \quad ; \quad \text { with } s \in\left[0, t_{\max }\right] .
$$

where $s$ scales the load level and plays a similar role to a pseudo-time, or arc-length parameter, for the particular designed trajectory.

The chosen network points, in this space of parameters, have coordinates taking three discrete values: $0,0.5$ and 1 , and therefore they constitute a hypercube in $\mathbb{R}^{4}$. The set of points generated by this combination is $3^{4}=81$. However, from this set, we remove the points staying on the same segment intersecting the origin because they define identical trajectories by re-scaling appropriately the parameter $s$. For example, once the point with coordinates $[1 ., 0,0,0]$ has been chosen, the point with coordinates $[0.5,0,0,0]$ is removed from the list. Removing these points from the list of the trained trajectories, the so-defined sampling program remains with 38 trajectories contributing to alleviate the involved off-line computational effort.

Furthermore, from every trained trajectory, we collect 70 snapshots of deformation gradient fluctuations $\chi_{\tilde{\boldsymbol{F}}_{\mu}}^{i}$, and 70 snapshots of internal free energy $\chi_{\varphi_{\mu}}^{i}$ in accordance with the following criterion, see also Figure 2-b:

1) ten equidistant snapshots are taken during the elastic micro-cell regime of each trained trajectory (all integration points of the micro-cell remain in the elastic regime $s \leq s_{I}$ ).

2) sixty equidistant snapshots are taken during the inelastic micro-cell regime of each trained trajectory (at least, one integration point of the micro-cell is in plastic regime $s \geq s_{I}$ ).

\section{Numerical Assessments of the HPROM Technique}

A ferritic ductile iron is adopted as the material to be simulated in the present numerical assessments. The micro-structure is represented by two components such as shown in Figure 3. A ferritic matrix with graphite nodules having a random distribution and sizes with average value of the order of $50 \mu \mathrm{m}$. This micro-structure is similar to that reported by [17], see also [18],

We assume that the matrix and the nodules have a perfect contact which is not degraded with the load increment. Graphite nodules are assumed hyperelastic in all cases. While the matrix should be 


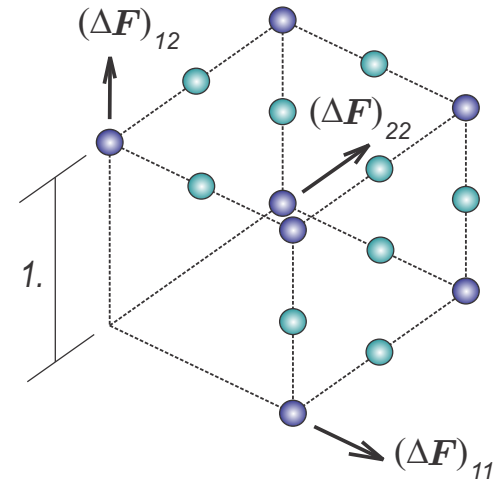

(a)

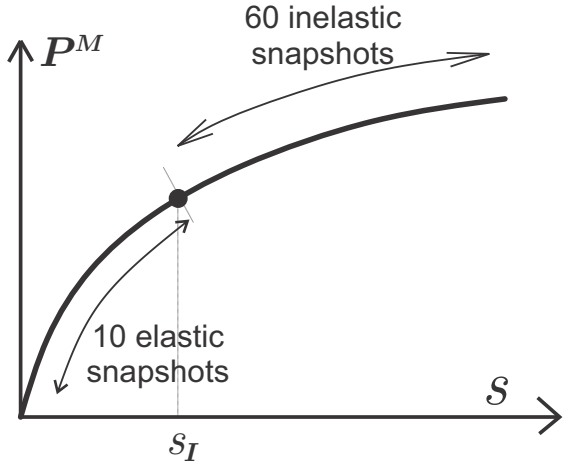

(b)

Figure 2: Parametrization of the micro-cell sampling program. The set of points constituting a network in $\mathbb{R}^{4}$ represents the set of trained trajectories. The coordinates of each network point define a trajectory with a deformation gradient increase being proportional to the coordinates of the corresponding network point. The magnitude of $\boldsymbol{F}^{M}$ is defined by the arc-length parameter $s$ in accordance with formula (38) : a) For visualization reasons, the network of points is depicted in a reduced $3 \mathrm{D}$ space by considering the components $\Delta F_{11}, \Delta F_{22}$ and $\Delta F_{12}$. b) Selection of elastic and inelastic snapshots during a sampled trajectory represented by the homogenized response: homogenized Piola-Kirchoff stress tensor $\boldsymbol{P}^{M}$ versus macro-deformation gradient .

considered hyperelastic in the numerical assessments addressed in sub-Section 5.2 and elasto-plastic in those addressed in sub-Section 5.3. The elastic and plastic free energy expressions utilized in each case are given by Eq. (A.1) and (A.6), in Appendix A, while the corresponding parameters are described in Table 2.

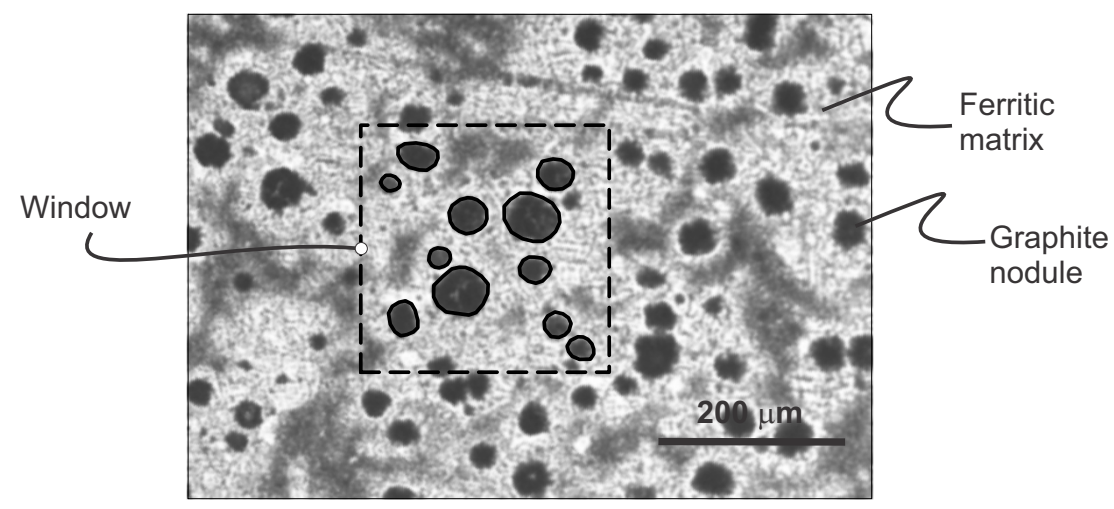

Figure 3: Material morphology and window utilized for the modeling.

\subsection{Micro-cell Model}

The window utilized for defining the micro-cell model is shown in Fig. 3. It does not matter to recognize if it constitutes an RVE. Here, instead, the attention is focused on testing three different finite element meshes modeling this window. The three meshes are displayed in Fig. 4 and are additionally described 
in Table 1. From the coarser to the finer meshes, they are denoted MI, MII and MIII, respectively. The finite element type utilized in the simulations is the one described in [19]. Periodic boundary conditions are imposed in the micro-cell finite element models such as described by [20].

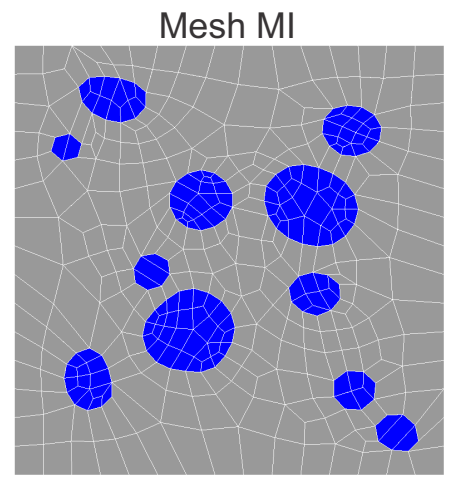

(a)

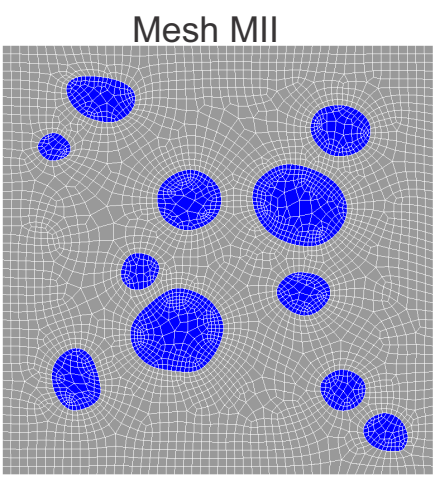

(b)

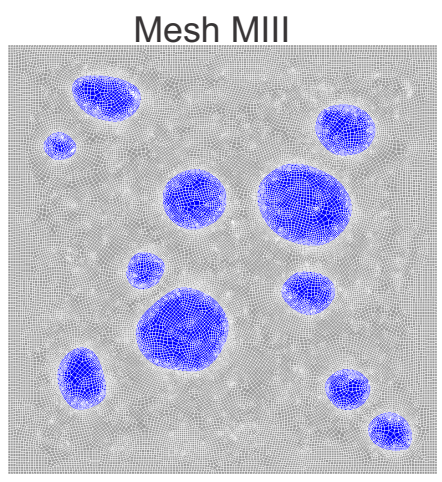

(c)

Figure 4: Microscale finite element models of a ferritic ductile iron: a) Mesh MI with 398 finite elements and 1592 quadrature points, b) Mesh MII with 4090 finite elements and 16360 quadrature points, c) Mesh MIII with 39970 finite elements and 159880 quadrature points.

\begin{tabular}{|c|c|c|c|}
\hline FE model & $\begin{array}{c}\text { Number } \\
\text { of elements }\end{array}$ & $\begin{array}{c}\text { Number } \\
\text { of d.o.f.'s }\end{array}$ & $\begin{array}{c}\text { Total number of } \\
\text { Gauss points }\left(N_{p g}\right)\end{array}$ \\
\hline M I & 398 & 858 & 1592 \\
M II & 4090 & 8382 & 16360 \\
M III & 39970 & 80610 & 159880 \\
\hline
\end{tabular}

Table 1: MI, MII and MIII finite element models.

The models denoted MIII-1 and MIII-2 use the same finite element mesh of model MIII. However, the material behavior for the ferritic (matrix) component in model denoted MIII-1 is assumed hyperelastic, i.e. $\sigma_{Y}^{0} \rightarrow \infty$, while the model MIII-2 is assumed to behave elasto-plastically.

\begin{tabular}{|l|c|c|c|c|c|c|}
\hline $\begin{array}{l}\text { Microscale } \\
\text { Component }\end{array}$ & $\begin{array}{c}E_{\mu} \\
{[\mathrm{GPa}]}\end{array}$ & $\nu_{\mu}$ & $\begin{array}{c}\sigma_{Y}^{0} \\
{[\mathrm{MPa}]}\end{array}$ & $\begin{array}{c}\Delta \sigma_{Y} \\
{[\mathrm{MPa}]}\end{array}$ & $\begin{array}{c}H_{\mu} \\
{[\mathrm{MPa}]}\end{array}$ & $\delta_{\mu}$ \\
\hline \hline metallic matrix & 200.0 & 0.30 & 10.0 & 0.265 & 0.12924 & 16.93 \\
\hline graphite inclusions & 30.0 & 0.20 & - & - & - & - \\
\hline
\end{tabular}

Table 2: Summary of material properties corresponding to the composite components: $E_{\mu}$ (Young's modulus), $\nu_{\mu}$ (Poisson ratio), $\sigma_{\mu}^{u}$ (ultimate tensile stress), $H_{\mu}$ (hardening modulus), $\sigma_{Y}^{0}$ (initial yield stress), $\Delta \sigma_{Y}$ (yield stress increment due to the exponential hardening law), $\delta$ (coefficient for the exponential hardening law, see Appendix A.

In this context, the accuracy of the ROM model mainly depends on the number of modes $n_{F}$ defining the basis $\{\boldsymbol{\Psi}\}$ of the micro-deformation gradient fluctuations field, see $[16,7]$; while the accuracy of the HPROM model also depends on the number of cubature points, $N_{r}$, adopted in the ROQ scheme. However, in [7], it has been shown a direct correspondence between $N_{r}$ and the number of energy modes 
$n_{\varphi}$ defining the basis $\{\boldsymbol{\Phi}\}$. In fact, it is: $N_{r}=n_{\varphi}+1$. Thus, the accuracy of the HPROM results will depend on both parameters, $n_{F}$ and $n_{\varphi}$.

In this section, a judicious analysis is performed to evaluate the sensitivity of the errors with respect to the parameters $n_{F}, n_{\varphi}$ and $N_{r}$, in different scenarios. Two different kinds of test are presented:

(1) Consistency tests: the goal is to analyze the errors introduced by the reduced models at reproducing trained trajectories used in the sampling program. The reference solutions are provided by the HFFEM. This kind of errors can be classified as truncation errors and sampling errors.

(a) Truncation errors: the POD technique selects a small number of modes from the snapshot matrices and neglects those associated with singular values smaller than a given threshold, and therefore, the reduced basis cannot span exactly all snapshots. This induces a truncation error which can be a-priori estimated with the singular value magnitudes of the neglected modes.

(b) Sampling errors: a second source of error is associated with the snapshot sampling technique. Only a few snapshots of each trained trajectory are taken to build the global snapshot matrices $\chi_{\tilde{\boldsymbol{F}}_{\mu}}$ and $\boldsymbol{\chi}_{\varphi_{\mu}}$. Therefore, the reduced basis cannot reproduce exactly the trained trajectory. This sampling error is evaluated a-posteriori requiring an on-line computation.

(2) Accuracy tests: the goal is to analyze the reduced model errors to reproduce random loading/unloading trajectories (different from the set of trained trajectories). Within the context of the present work, these tests can be performed either by injecting a macro-deformation gradient, different to the ones used in the sampling program, or alternatively, by solving a full multiscale problem, where the macro-deformation gradient, acting on the micro-cell, is obtained from the resolution of the macro-scale equilibrium problem.

In accordance with this classification of the ROM errors, we present the following numerical results in three parts. The first two parts, sub-sections 5.2 and 5.3, are devoted to study the consistency of the methodology, for both, hyperelastic (sub-section 5.2) and elastoplastic (sub-Sections 5.3) materials, with the ROM and HPROM formulations. The third part, section 5.4, is devoted to studying the accuracy error of the HPROM formulation simulating a full multiscale test.

\subsection{Numerical Assessment of HPROM Techniques for Hyperelastic Materials}

In this first case, a hyperelastic material is used with the MIII-1 model. Material properties are presented in Table 1 and Table 2.

Three trajectories, namely A, B and C, have been taken from the sampling program to check the consistency of the methodology. In accordance with Eq. (38), they are defined by the following parameters

$$
\begin{array}{cc}
\text { Trajectory A : } & \left(\Delta F_{11}, \Delta F_{12}, \Delta F_{21}, \Delta F_{22}\right)=(0.5,1,0,0) ; \\
\text { Trajectory B : } & \left(\Delta F_{11}, \Delta F_{12}, \Delta F_{21}, \Delta F_{22}\right)=(0,0.5,1,0) ; \\
\text { Trajectory C : } & \left(\Delta F_{11}, \Delta F_{12}, \Delta F_{21}, \Delta F_{22}\right)=(1,0.5,1,1) ; \\
& \text { and } \quad s \in[0,0.6] .
\end{array}
$$


Figure 5 displays the colour maps of the $\tilde{\boldsymbol{F}}$-components corresponding to trajectory B at the end of the analysis, $s=0.6$, obtained with the HFFEM. Notice the maximum values attained in this solution, as well as the highly nonuniform distribution across the micro-cell domain.

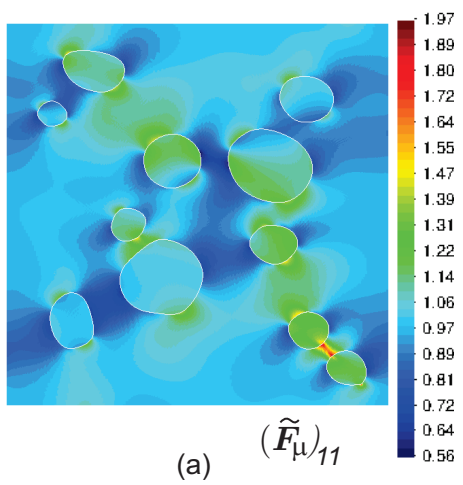

(a)

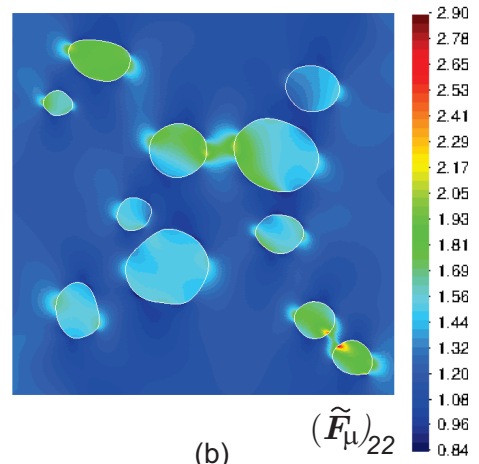

(b)

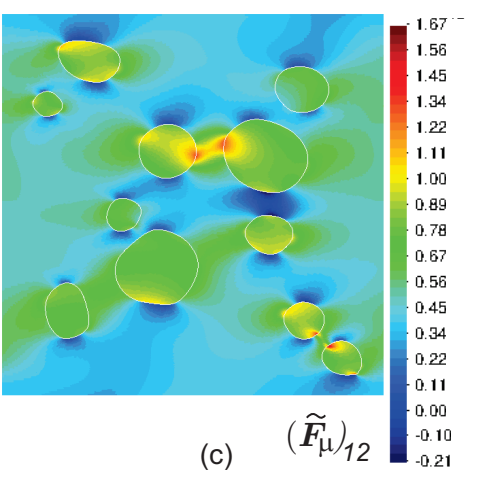

(c)

Figure 5: Colour maps of $\tilde{\boldsymbol{F}}_{\mu}$ at the end of analysis, trajectory B, MIII-1 model (39970 finite elements): (a) $\left(\tilde{\boldsymbol{F}}_{\mu}\right)_{11}$, (b) $\left(\tilde{\boldsymbol{F}}_{\mu}\right)_{22},(\mathrm{c})\left(\tilde{\boldsymbol{F}}_{\mu}\right)_{12}$. HFFEM solution.

\subsubsection{Consistency Tests}

\section{a) Singular Value Spectrum}

The Singular Value Decomposition (SVD) of a snapshot matrix provides the spectrum of singular values which are related to each orthonormal mode. This spectrum gives an a-priori estimation of the truncation error of the removed modes when the reduced basis is chosen.

Fig. 6 presents the singular value spectrum of the snapshot matrices $\chi_{\tilde{\boldsymbol{F}}_{\mu}}$ and $\chi_{\varphi_{\mu}}$ that have been obtained with the above described sampling program. A similar decreasing tendency is observed for both spectra.

In order to guarantee a proper development of the present methodology, it is recommended to exclude from the reduced basis the modes associated with very low singular values.

\section{b) A-priori ROM Errors}

Once the the POD basis $\{\boldsymbol{\Psi}\}$ of the reduced space for the deformation gradient fluctuation has been built, the a-priori error to retrieve a snapshot $\chi_{\tilde{\boldsymbol{F}}_{\mu}}^{i}$ can be assessed through

$$
\operatorname{error}_{\Psi}^{i}=\frac{\left\|\chi_{\tilde{\boldsymbol{F}}_{\mu}}^{i}-\boldsymbol{\Psi} \boldsymbol{c}\right\|}{\left\|\chi_{\tilde{\boldsymbol{F}}_{\mu}}^{i}\right\|}
$$

where the vector $\boldsymbol{c}$ collects the linear combination coefficients of the vector $\chi_{\tilde{\boldsymbol{F}}_{\mu}}^{i}$ projected onto the space spanned by $\{\boldsymbol{\Psi}\}$. Clearly, if the base $\{\boldsymbol{\Psi}\}$ span the full space of the snapshot matrix (i.e. all modes 


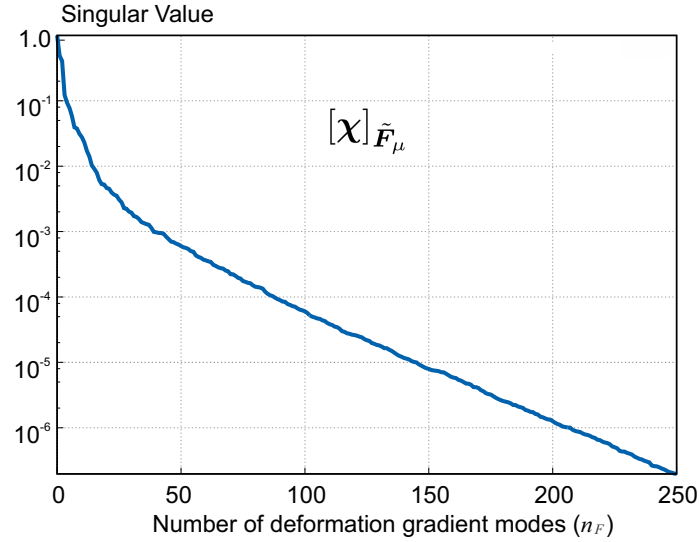

(a)

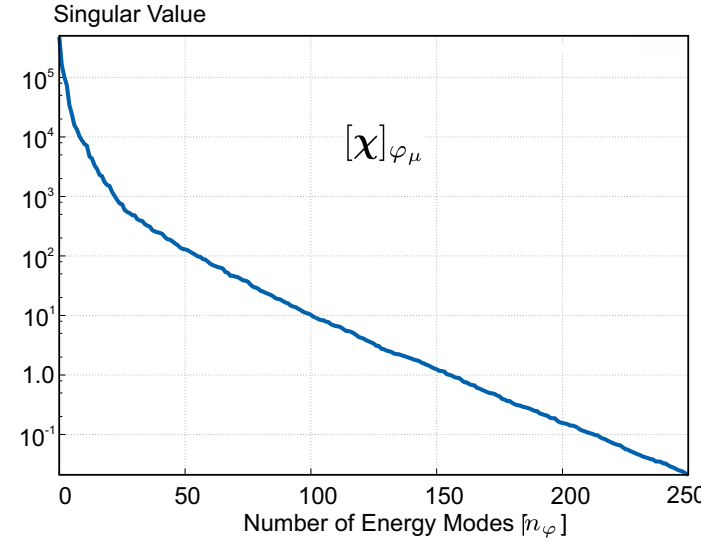

(b)

Figure 6: Singular value spectra of the snapshot matrices: a) $\chi_{\tilde{\boldsymbol{F}}_{\mu}}$ and; b) $\chi_{\varphi_{\mu}}$. MIII-1 model (39970 finite elements)

determined by the SVD of the snapshot matrix are included in the basis), the error in Eq. (39) is zero. The truncation error is given by

$$
\operatorname{Error}_{\Psi}=\max _{i}\left(\operatorname{error}_{\Psi}^{i}\right) \quad ; \quad \text { for } i=1, \ldots, N_{\text {snp }}
$$

An identical a-priori truncation error, Error $\boldsymbol{\Phi}$, can be computed for the snapshot matrix $\boldsymbol{\chi}_{\varphi_{\mu}}$ using the energy reduced base $\{\boldsymbol{\Phi}\}$.

Both truncation errors, Error $\boldsymbol{\Psi}$ and Error $_{\boldsymbol{\Phi}}$, as functions of the number of modes defining the corresponding basis have been computed for trained trajectories A, B and C. These errors are shown in Fig. 7. Notice that the error responses changes slightly with different trajectories and they are nearly zero with a large enough number of modes.

The remarkable point that must be analyzed in plots of Fig. 7, corresponds to the rate at which this error goes to zero. Indeed, very steep curves in the region with a low number of modes, identify an optimal reduction procedure requiring a low quantity of modes to satisfactorily approach the solution field.

\subsubsection{Accuracy Tests}

a) A-posteriori ROM errors

The following relative percentage measure is proposed to analyze the a-posteriori ROM errors

$$
\text { Error }_{\boldsymbol{P}}[\%]=\frac{\int_{0}^{t_{\max }}\left\|\boldsymbol{P}_{R O M}^{M}-\boldsymbol{P}_{H F}^{M}\right\| d s}{\int_{0}^{t_{\max }}\left\|\boldsymbol{P}_{H F}^{M}\right\| d s} \times 100
$$

where $\boldsymbol{P}_{R O M}^{M}$ and $\boldsymbol{P}_{H F}^{M}$ are the First Piola-Kirchoff homogenized (macro) stress tensors computed with the ROM and HFFEM, respectively. Fig. 8-a compares the a-posteriori errors attained with this 


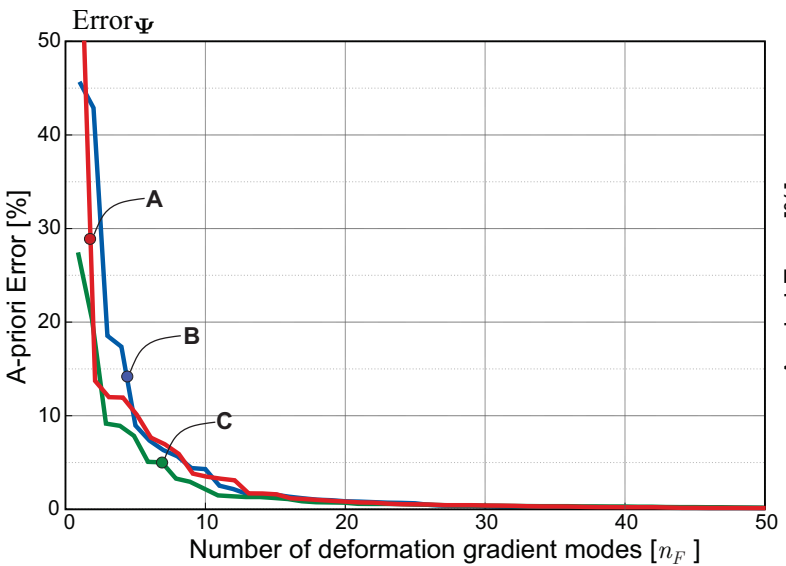

(a)

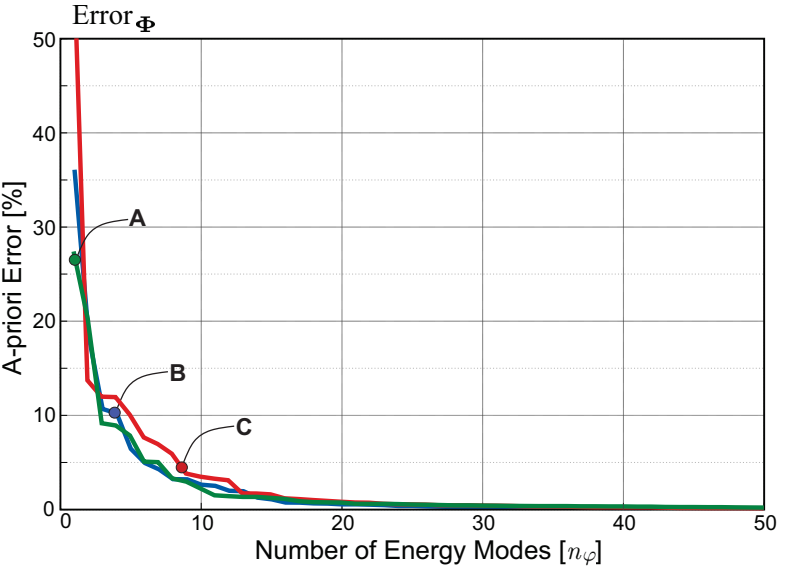

(b)

Figure 7: Consistency test: A-priori consistency error Error $_{\Psi}$ and Error $_{\boldsymbol{\Phi}}$, as functions of the number of modes defining the basis $\{\boldsymbol{\Psi}\}$ and $\{\boldsymbol{\Phi}\}$, respectively. Model MIII-1, subjected to three different training trajectories, A, B and C: (a) Error $_{\boldsymbol{\psi}}$, (b) Error $_{\boldsymbol{\phi}}$

criterion, with the MIII-1 model and simulating the sampled trajectories A, B and C. While, Fig. 8-b shows the error attained by simulating an arbitrary non-trained trajectory. Convergence toward the HFFEM solution is achieved when the number of deformation gradient modes $\left(n_{F}\right)$ is increased.

In addition, it can be noticed that taking $n_{F}=20$, regardless of the trajectory reproduced with the ROM model, the a-posteriori error is close to zero. So, this analysis is appropriated to check the quality of the sampling program to explore the full space of deformation gradient fluctuations. Being also used to define a lower bound in the number of modes for computing the online stage.

Fig. 9 plots the homogenized response $\left(\boldsymbol{P}^{M}\right.$ vs. $\left.\boldsymbol{F}^{M}\right)$ provided by the ROM model, with an increasing number of deformation gradient modes, $n_{F}$, for trajectory B. From these results, it is checked that both axial components of $\boldsymbol{P}_{R O M}$ match the homogenized solution of the HFFEM when the deformation gradient modes in the corresponding POD basis is increased.

\section{b) HPROM solutions for sampled trajectories}

The plots in Fig. 10 display the homogenized First Piola-Kirchoff stress tensor components $(\boldsymbol{P})_{11}$ and $(\boldsymbol{P})_{22}$ obtained with HPROM vs. the load level of the trained trajectory B. At the same time, these results are compared with the HFFEM solution. The HPROM solutions are obtained with two number of deformation gradient modes, $n_{F}=5$ and $n_{F}=10$, respectively, combined with a number of reduced order quadrature points $N_{r}$.

The plots in Fig. 11 depict the relative percentaje error, $\operatorname{Error}_{\boldsymbol{P}}[\%]$, , defined by Eq. (41) and evaluated with the homogenized stress tensor $\boldsymbol{P}_{H P R O M}^{M}$ instead of $\boldsymbol{P}_{R O M}^{M}$, versus the number of quadrature points $N_{r}$ utilized by the ROQ scheme. These errors are plotted using $n_{F}=10$ and $n_{F}=12$ deformation 


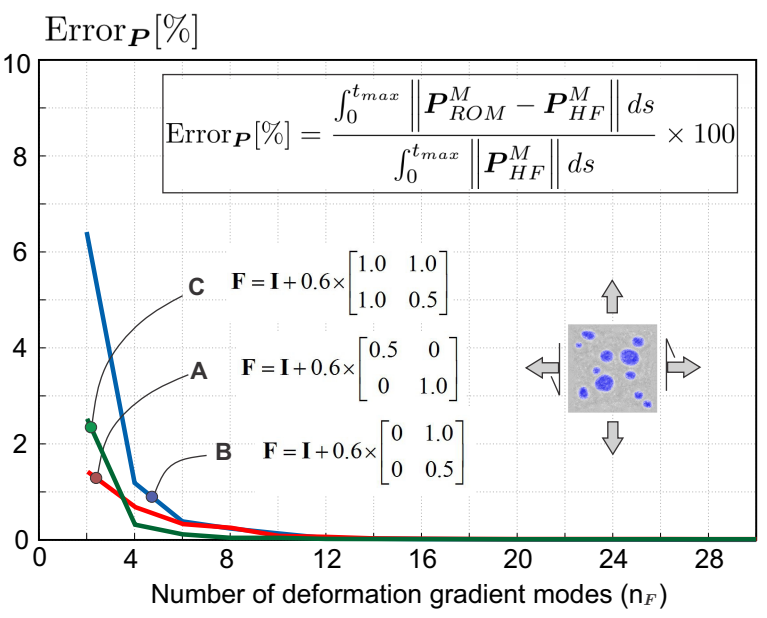

(a)

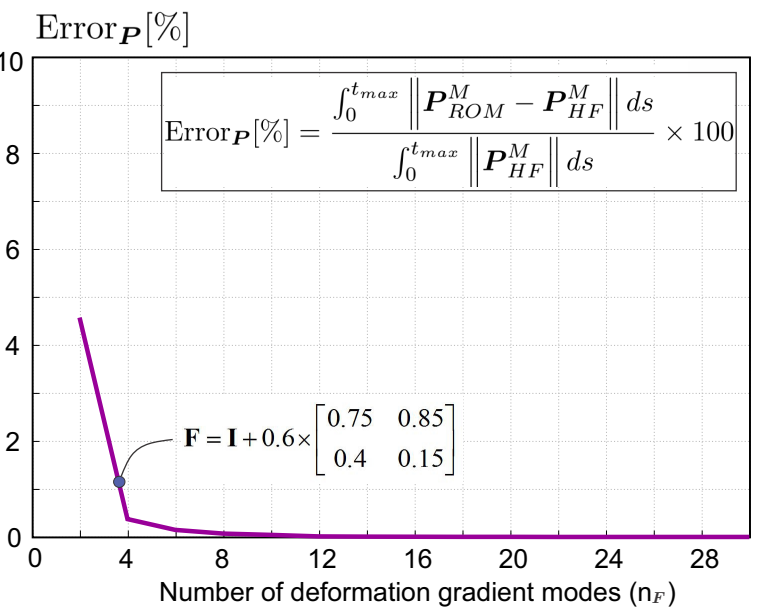

(b)

Figure 8: Consistency test: Analysis of results obtained with the ROM model. Error in the homogenized First PiolaKirchoff stress tensor vs. number of deformation gradient modes at the end of the trained trajectories. $\left(\boldsymbol{P}_{R O M}^{M}\right.$ and $\boldsymbol{P}_{H F}^{M}$ are the homogenized stresses obtained with the ROM and HFFEM, respectively). (a) Results obtained with three different trained trajectories: A, B and C with MIII-1 model. (b) Results obtained for an arbitrary (non-trained), trajectory.

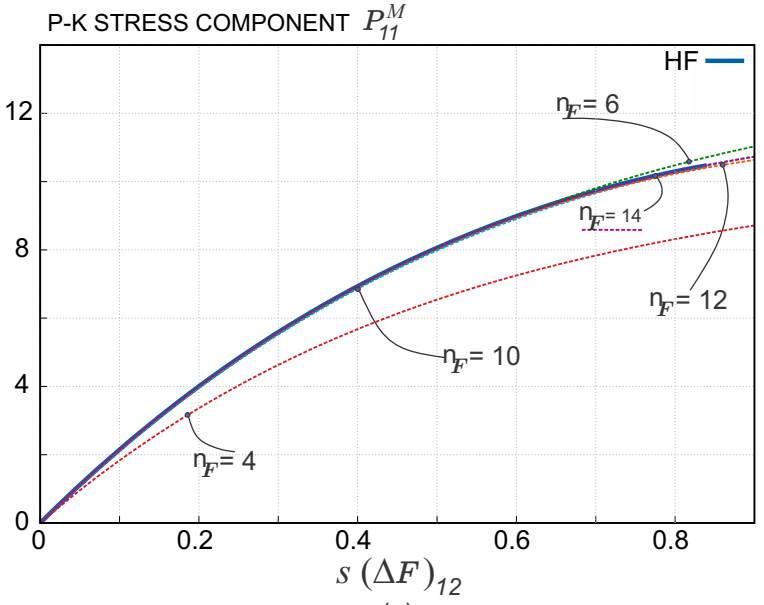

(a)

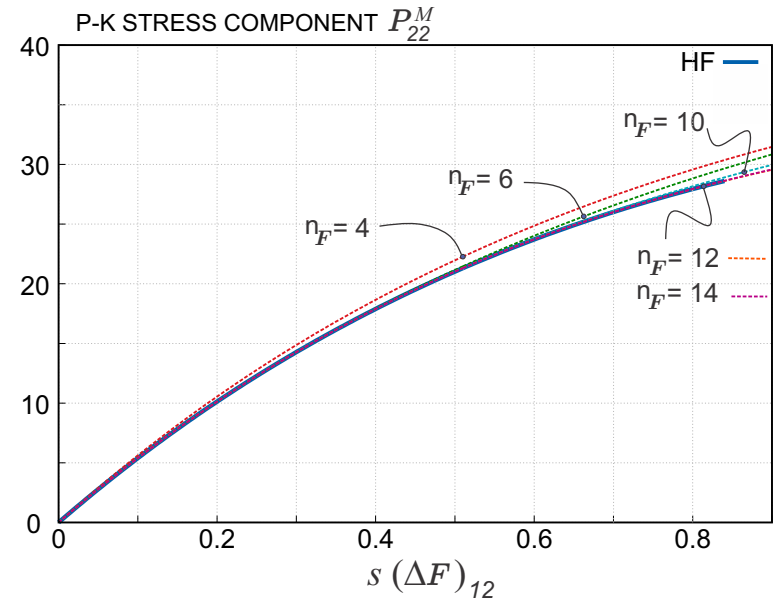

(b)

Figure 9: Accuracy test: ROM homogenized stress components in terms of the gradient fluctuations modes $\left(n_{F}\right)$ for trained trajectory B. Results are compared with the HFFEM solution obtained with the MIII-1 model (39970 finite elements): a) $\boldsymbol{P}_{11}^{M}$ vs. $s(\Delta F)_{11}$; b) $\boldsymbol{P}_{22}^{M}$ vs. $s(\Delta F)_{22}$. Curves denoted HF are obtained with the HFFEM. 


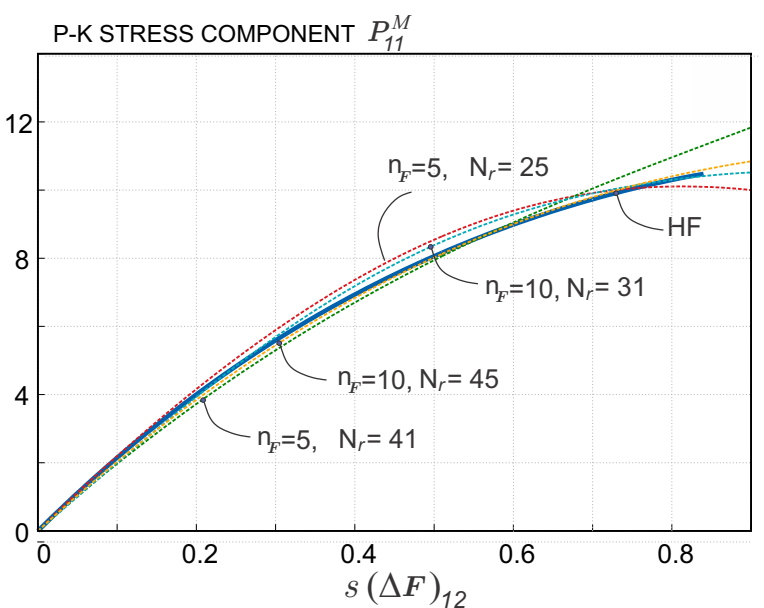

(a)

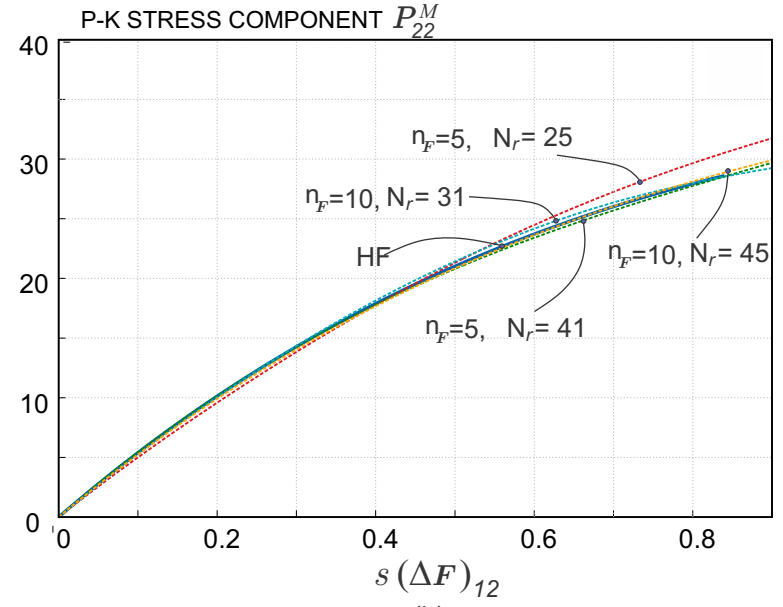

(b)

Figure 10: Accuracy test: HPROM homogenized stress components in terms of the number of quadrature points $N_{r}$ of the ROQ scheme for training trajectory B, using Model MIII-1 with 39970 finite elements: a) $\boldsymbol{P}_{11}^{M}$ vs. $s(\Delta F)_{11} ;$ b) $\boldsymbol{P}_{22}^{M}$ vs. $s(\Delta F)_{22}$. Curves denoted HF are obtained with the HFFEM.

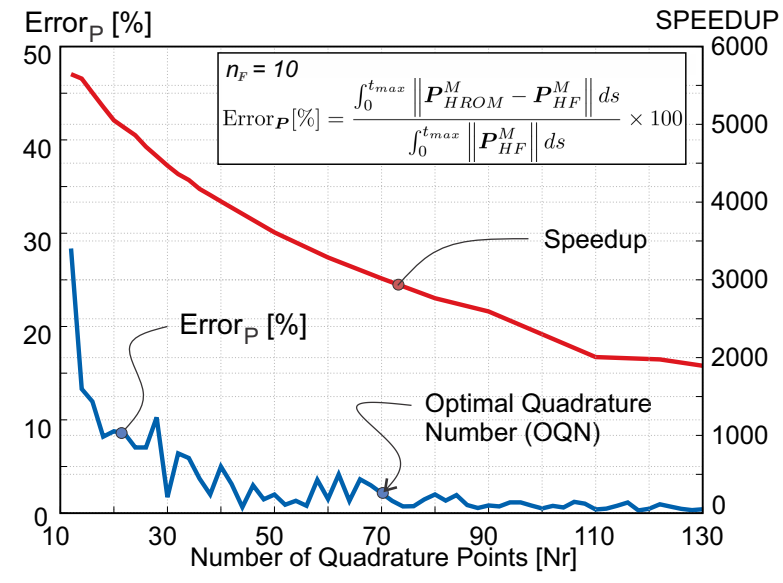

(a)

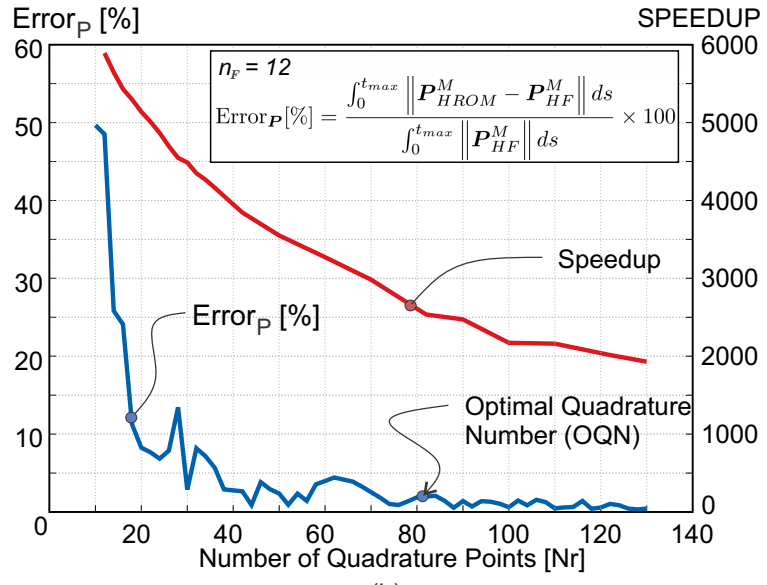

(b)

Figure 11: Accuracy analysis of the HPROM results. MIII-1 model, original HFFEM mesh with 39970 finite elements. (a) Macro-stress errors and Speed-ups vs. number of quadrature points $\left(N_{r}\right)$ of the ROQ scheme, obtained for $n_{F}=10$.

(b) Macro-stress errors and Speed-ups vs. number of quadrature points $\left(N_{r}\right)$ of the ROQ scheme, obtained for $n_{F}=12$. The Optimal Quadrature Number (OQN) is shown in both cases. 
gradient modes. In the same plots, the computational speed-up ${ }^{5}$, is also depicted. Noticed that for very low values of $N_{r}$, the solution of the HPROM is unstable and noisy. However, by selecting a large enough value of $N_{r}$, the error holds small and almost constant, indicating the existence of a minimum amount of quadrature points, called the Optimal Quadrature Number (OQN). This threshold value is considered an optimum number because a significant increase of quadrature points does not provide a notably increase in the accuracy. Therefore, the OQN must be used in order to guarantee an optimal performance of the HPROM model.

\subsubsection{Design Strategy for Hyperelastic Materials}

Performing the same procedure to that used for obtaining the results of Fig. 11, for a number of $n_{F}$ and $N_{r}$, we can collect all results and build the abacus displayed in Fig. 12. The upper plot in this Figure gives the $\operatorname{Error}_{P}[\%]$ vs. $n_{F}$, while the lower plots display the OQN and speed-ups vs. $n_{F}$. This abacus is valid for model MIII-1.

Both plots in Fig. 12 can be used as an adequate tool for an a-piori design of the HPROM strategy in the following sense: the methodology begins by selecting the admissible error (e.g. $<1.0 \%$ ), then, in accordance with the abacus, the amount of modes needed to obtain this error is $n_{F}=12$. Taking this value of $n_{F}$, and entering to the lower graph in Fig. 12, an optimal number of quadrature points $(O Q N=75)$ is obtained, with a corresponding speed-up of $\sim 2700$.

The availability of such an abacus (a-priori constructed) for a specific RVE microstructure, allows the user's selection of the most appropriate HPROM strategy, by balancing the admissible error vs. the desired speed-up, to guarantee its optimal performance in the on-line computational stage.

\subsection{Numerical Assessment of HPROM Techniques for Elasto-plastic Materials}

The following tests are performed by modeling the matrix of the composite in Fig. 3 with and elastoplastic constitutive model and the graphite nodules with an hyperelastic model. The elastic and plastic free energy expressions for both material models are given by Eq. (A.1) and (A.6), in Appendix A, while the corresponding parameters are described in Table 2.

Next, the uni-axial trajectory with macro-deformation gradient defined according to Eq. (38) and

$$
\left(\Delta F_{11}, \Delta F_{22}, \Delta F_{12}, \Delta F_{21}\right)=(1,0,0,0)
$$

is used to check the consistency of the methodology. With this trajectory, it can be reached a highly non-uniform pattern of strain distribution without inducing loss of macro-stability.

Fig. 13 displays the color maps of the deformation gradient fluctuation components obtained with the HFFEM. We analyze the capacity of the HPROM strategy for capturing this rather complex deformation pattern observed in previous stages to the detection of macroscopic bifurcation.

\footnotetext{
${ }^{5}$ Speed-up are evaluated as the ratio between time required to compute the HFFEM solution and the time required to compute the reduced model solution.
} 

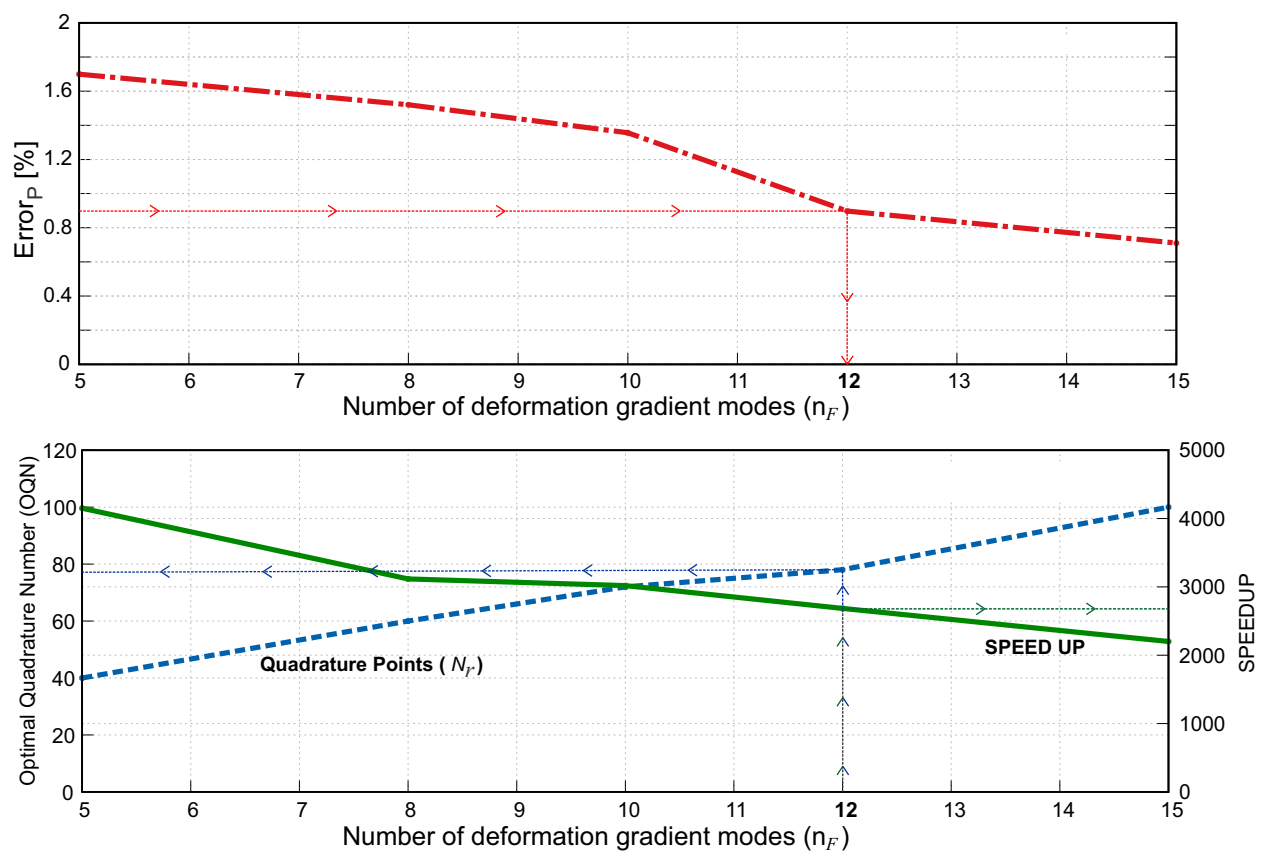

Figure 12: Design diagrams for the HPROM strategy, hyperelastic material. Top: HPROM (stress) errors in terms of the number of deformation gradient modes. Bottom: OQN and obtained speed-up in terms of the number of deformation gradient modes. By selecting the admissible error (say 0.9\%) in the upper diagram, one obtains the requested number of strain modes, $n_{F}=12$. Entering with this result in the lower diagram, one obtains the optimal number of quadrature points $(\mathrm{OQN}=78)$ and the resulting speed-up (speed-up approx. 2700).

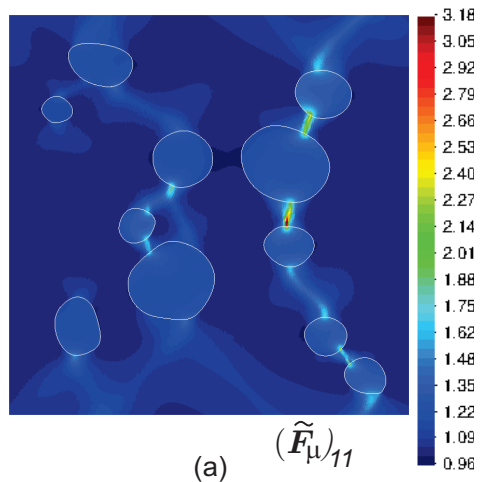

(a)

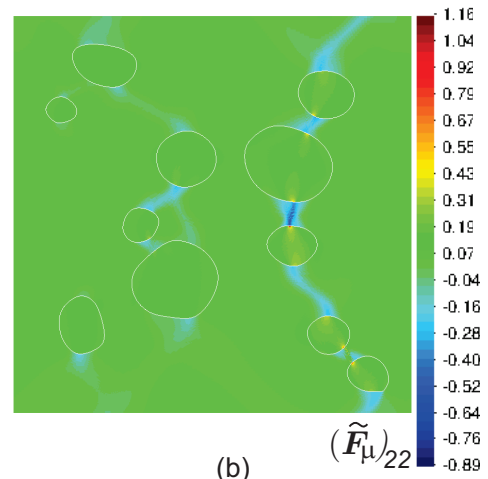

(b)

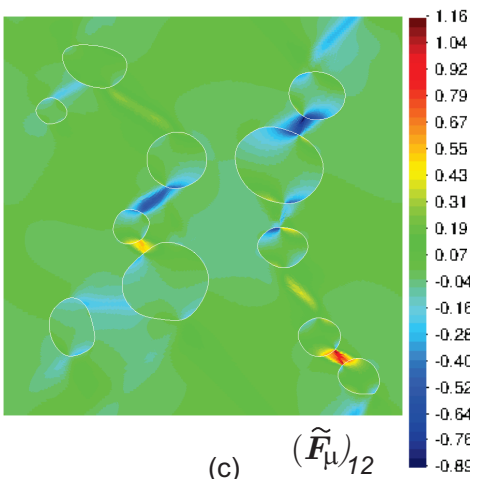

(c)

Figure 13: Color maps of the deformation gradient fluctuation field, MIII-2 model (39970 finite elements): (a) Component $\left(\tilde{\boldsymbol{F}}_{\mu}\right)_{11}$, (b) Component $\left(\tilde{\boldsymbol{F}}_{\mu}\right)_{22}$, (c) Component $\left(\tilde{\boldsymbol{F}}_{\mu}\right)_{12}$. Solution obtained wi the HFFEM. 


\subsubsection{Singular Value Spectrum}

The singular value spectra resulting from the SVD of the snapshot matrices $\chi_{\tilde{\boldsymbol{F}}_{\mu}}$ and $\boldsymbol{\chi}_{\varphi_{\mu}}$ are depicted in Fig. 14. Comparing these spectra with those of Fig. 6, it can be concluded that, a larger amount of modes are needed by the elasto-plastic model to decrease the truncation errors at a given order of magnitude.

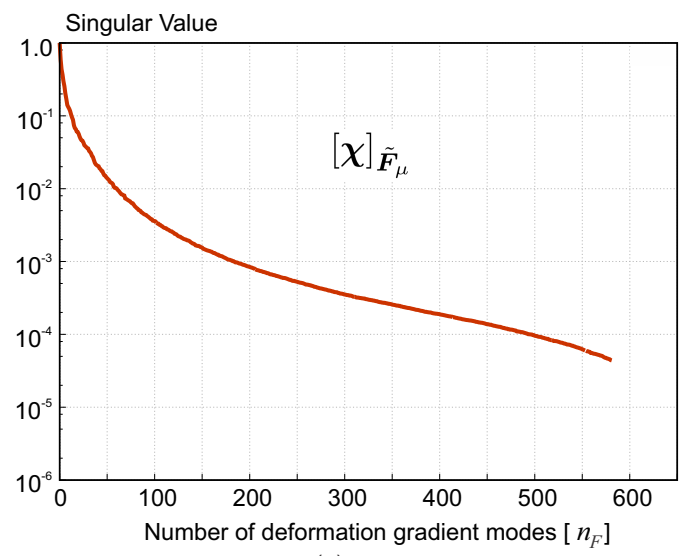

(a)

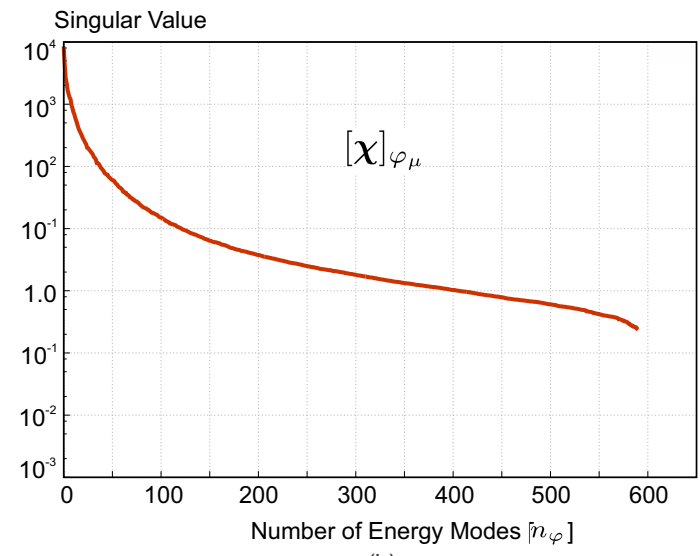

(b)

Figure 14: Singular value spectra resulting from the SVD of the deformation gradient fluctuation and internal free energy matrices, MIII-2 model (39970 finite elements).

\subsubsection{HPROM Solutions of Sampled Trajectories}

The accuracy of the HPROM strategy for capturing the uni-axial sampled trajectory is assessed in Fig. 15. The plots in this Figure display the homogenized First Piola-Kirchoff stress tensor components $\left(\boldsymbol{P}^{M}\right)_{11}$ and $\left(\boldsymbol{P}^{M}\right)_{22}$ obtained with HPROM vs. the corresponding deformation gradient increase. Several plots, with two different sets of deformation gradient modes: $n_{F}=30$ and $n_{F}=40$, show the sensitivity of the homogenized stresses with the increase of $N_{r}$. Curves denoted HF are obtained with the HFFEM.

As anticipated above, in contrast with the hyperelastic case, the elasto-plastic case needs a larger amount of deformation gradient modes to accurately match the HFFEM solution. In consequence, a larger number of reduced integration points must also be used to obtain an accurate response.

The plots in Fig. 16 display the error of the homogenized stress tensor evaluated with the HPROM model by changing the number of quadrature points $N_{r}$. They have been obtained with $n_{F}=40,50$ and 60 deformation gradient modes. In the same plots, the computational speed-up with respect to the $\mathrm{HF}$ case is also depicted. Note that, by selecting a high enough value of $N_{r}$, the error holds small and almost constant. Indicating, once again, the existence of an Optimal Quadrature Number (OQN).

In the same Fig. 16, it can be observed a residual error of the order of $2 \%$, even for large values of $n_{F}$. This residual error is associated with a sampling error, which could be diminished by increasing the number of snapshots taken from the uni-axial trajectory to compute the reduced bases. 


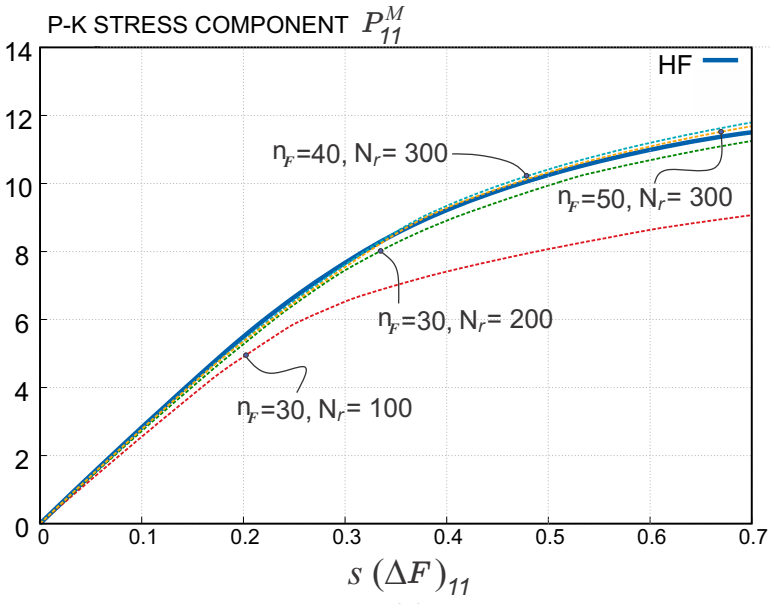

(a)

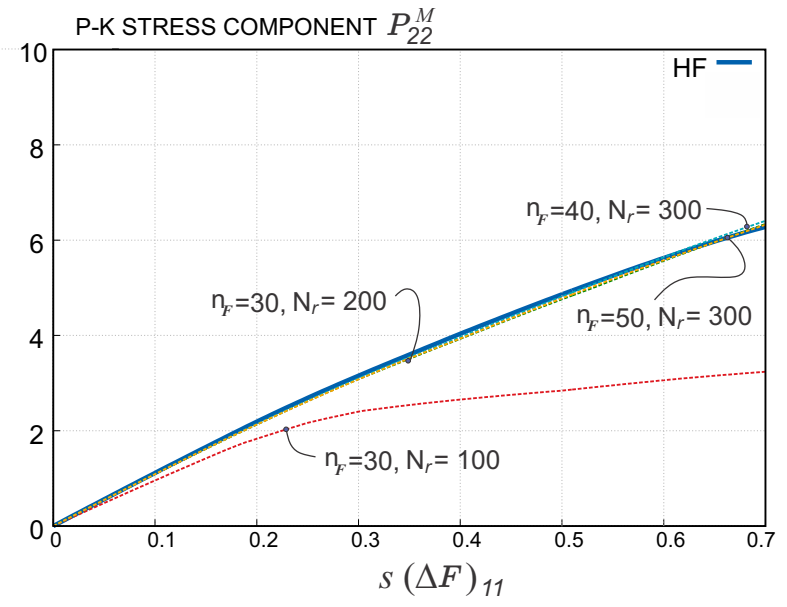

(b)

Figure 15: Accuracy test: First Piola-Kirchoff stress components $\left(\boldsymbol{P}^{M}\right)_{11}$ and $\left(\boldsymbol{P}^{M}\right)_{22}$ computed with the HPROM strategy, with the trajectory defined by an uni-axial deformation gradient, using MIII-2 model (39970 finite elements). Curves denoted HF have been obtained with the HFFEM.

\subsubsection{Design Strategy}

Fig. 17 shows the summary of results obtained with the HPROM strategy, using a wide range of deformation gradient modes, with MIII-2 model. The Optimal Quadrature Number for all set of deformation gradient modes is also depicted.

This Figure can also be used to obtain a HPROM design strategy for the elasto-plastic MIII model. In this case, the strategy is similar to that described for hyperelastic materials in sub-section 5.2.3. By assuming an acceptable maximum error, the upper plot in Fig. 17 gives the number of gradient deformation fluctuation modes that, at least, should be utilized. Once the number of modes $n_{\boldsymbol{F}}$ has been defined, Fig. 17-b provides the number $N_{r}$ which should, at least, used to obtain the corresponding speed-up (dark green line). We recall that the number of quadrature points $N_{r}$ is intimately associated with the number of energy modes $\left(n_{\varphi}=N_{r}-1\right)$.

\subsubsection{Speed-up Scalability}

An issue of primary importance in developing a successful HPROM technique is related to the expected performance trend, in terms of speed-up, when the HFFEM increases its complexity. The notion of complexity refers to the problem size, here evaluated in term of d.o.f.'s, of the HFFEM.

We evaluate this issue for the present HPROM technique. To perform this analysis, we solve the trajectory with a given macro-deformation gradient that corresponds to a uni-axial stretching $(\Delta \boldsymbol{F}=$ $[1,0,0,0])$, using the elasto-plastic material described in the previous subsections, with three finite element meshes denoted MI, MII and MIII, respectively.

The optimal selection of $n_{F}$ and $O Q N$, for the three models, have been carried out by using a similar abacus to that of Fig. 17, and with the objective that the Error $[$ [\%] does not exceed $2.5 \%$ for each model. 


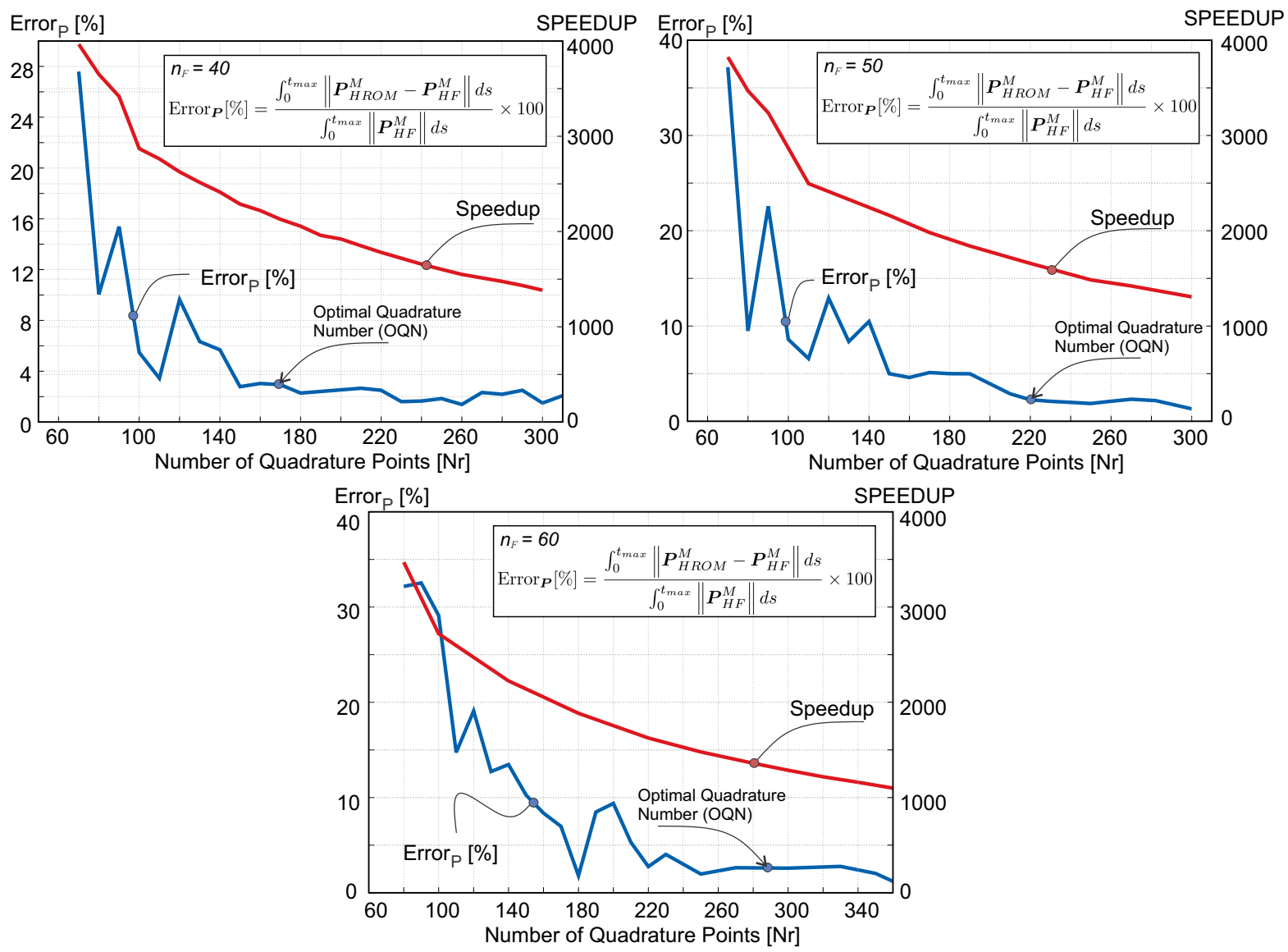

Figure 16: Accuracy test: results obtained with the HPROM model in terms of the number of quadrature points $\left(N_{r}\right)$ of the reduced integration scheme. Error and Speed-up obtained for: (a) $n_{F}=40$. (b) $n_{F}=50$. (c) $n_{F}=60$. The Optimal Quadrature Number (OQN) is shown in all cases. MIII-2 original HFFEM with 39970 finite elements. 

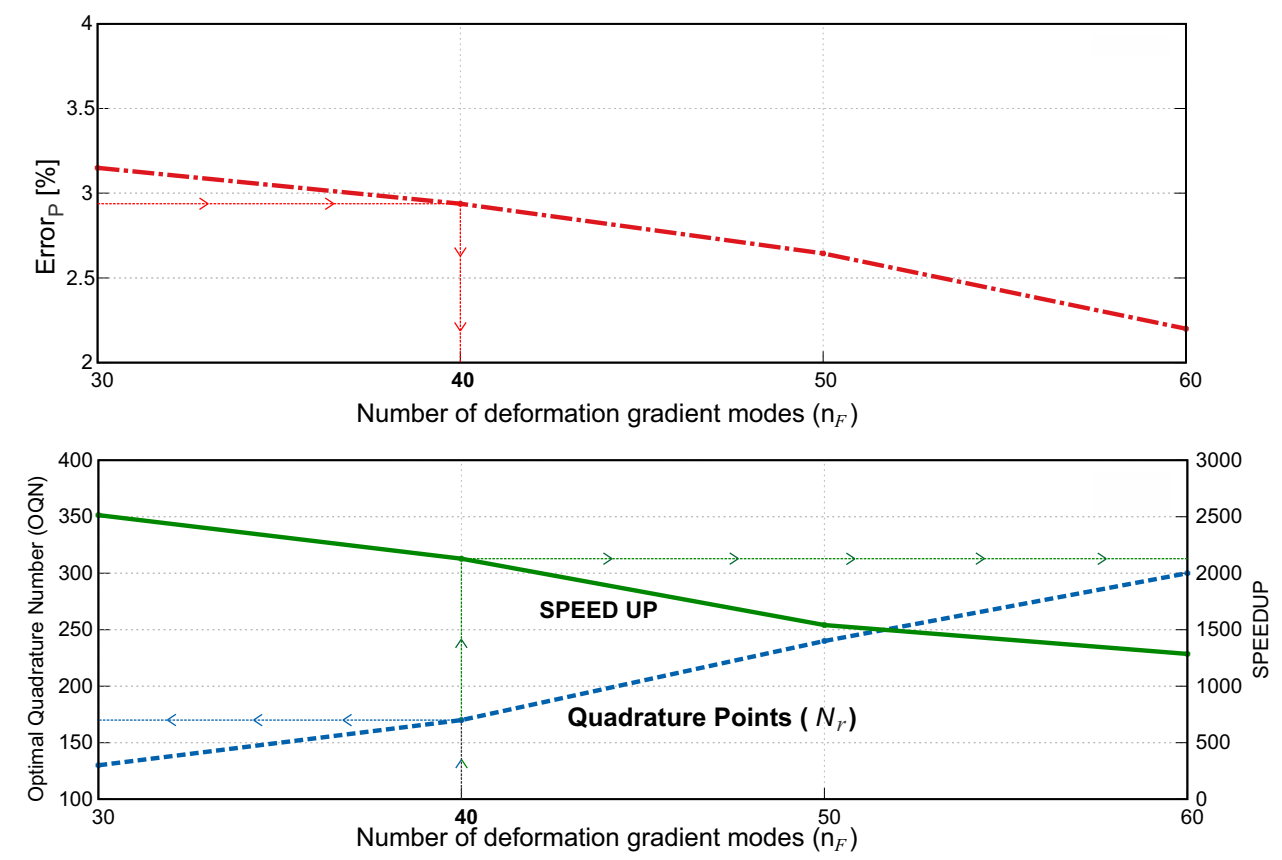

Figure 17: HPROM design diagrams. Top: HPROM error in terms of number of strain modes. Bottom: OQN and obtained speed-up in terms of the number of strain modes. By selecting the admissible error (say 3.0\%) in the upper diagram, one obtains the requested number of deformation gradient modes, $n_{\varepsilon}=40$. Entering with this result in the lower diagram one obtains the suitable number of integration points $(\mathrm{OQN}=175)$ and the resulting speed-up (speed-up $=2100)$. 
The speed-ups are computed for the three HPROM cases. The obtained results in terms of speed-ups vs. HFFEM complexity (total number of d.o.f.'s) are plotted in a log-log curve depicted in Fig. 18. The same Figure also displays the real computational times required for each HPROM to solve one time step.

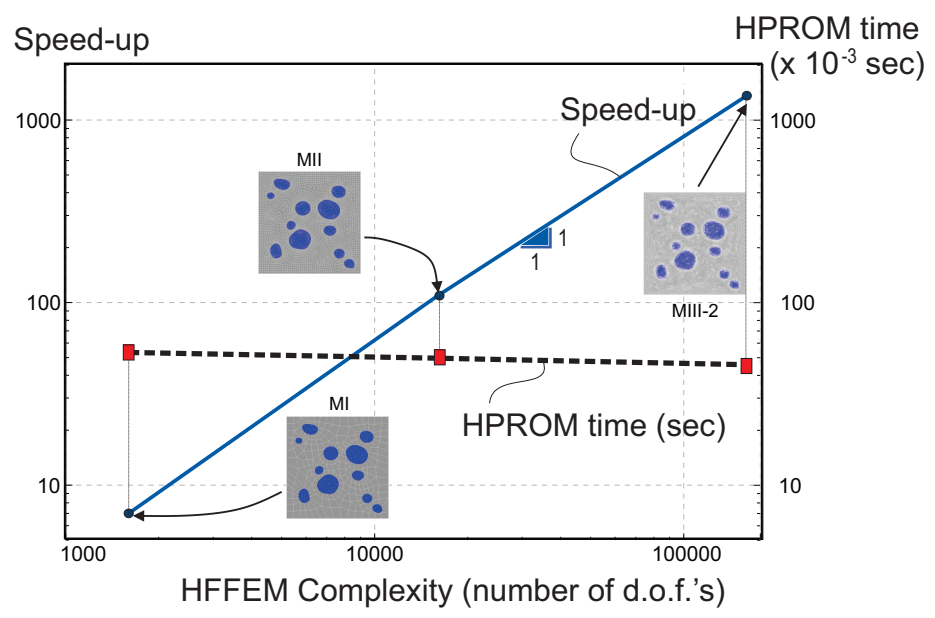

Figure 18: Scalability of the HPROM methodology. Speed-ups vs. HFFEM complexity and HPROM real computational time to compute one time step.

Remark: The linear log-log scalability character displayed in Fig. 18 can be justified from the following facts:

1) for the same micro-cell morphology, with fine enough finite element meshes, the required modes for approaching the corresponding deformation gradient fluctuations and energies tend to be the same, independently of the mesh complexity;

2) therefore, the computational cost of the HPROM is going to be very similar "disregarding the discretization level of the HFFEM mesh";

3) in consequence, the HPROM speed-up grows with the HFFEM cost of the micro-cell analysis at the same rate in terms of the complexity. Therefore, in terms of the complexity, "the speed-up increase follows the same law as the HFFEM computational cost increase".

\subsection{Accuracy Test: Multiscale Test}

Next, we present a numerical evaluation of a structural bar constituted by the same composite shown in Fig. 3, using a two-scale approach.

The main objective of this section is to evaluate the HPROM methodology and compare its results with the HFFEM, in terms of accuracy and speed-ups.

The bar is stretched along its axis. The geometry and boundary conditions of the simulated specimen are depicted in Fig. 19. Plane strain assumption is adopted and the thickness is $0.001 \mathrm{~m}$. 
The bar domain is split into two regions. 1) The multi-scale region with 190 finite elements at the macro-scale. Full geometrical and material non-linearies are simulated at this region. The MII microcell model, shown in Fig. 4, is used for simulating the micro-structure. 2) The remaining parts of the metal bar are modeled with an elastic monoscale approach (using 96 finite elements). The elasticity tensor in this region is obtained through an homogenization of the micro-structure elastic properties in the central bar region.

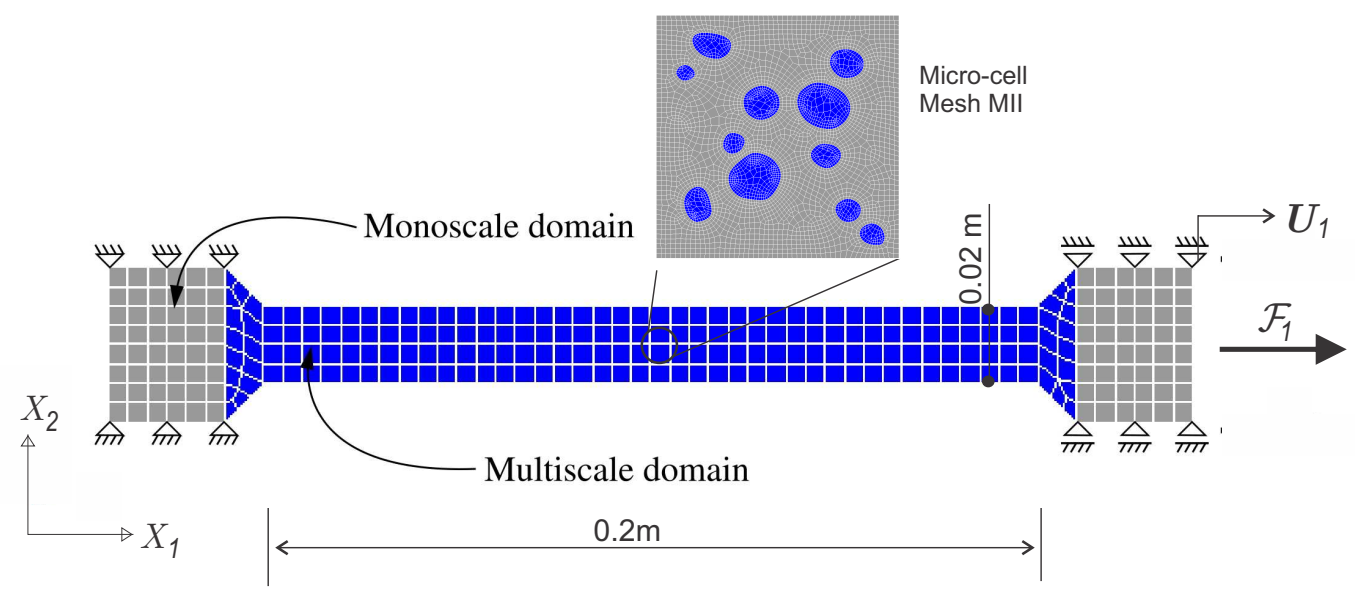

Figure 19: FE Mesh and boundary conditions for the macro-scale specimen of the multiscale analysis.

\subsubsection{HPROM Solutions}

Figure 20 plots the macro-structural response in terms of force, $\mathcal{F}_{1}$, vs. displacements $\boldsymbol{U}_{1}$ imposed at the end of the bar. The bar is stretched up to $17.5 \%\left(U_{1}=0.035 \mathrm{~m}\right)$. The plots compare the results obtained with the HFFEM response and three HPROM simulations, assuming the same number of deformation modes $\left(n_{F}=30\right)$ in the three simulations. The quantity of quadrature points $N_{r}$ are depicted for each plot.

In addition, in the same Figure 20, we show the relative errors of the computed structural response for each HPROM solution. The relative errors have been computed as follows

$$
\text { error }=\int_{\boldsymbol{U}_{1}=0}^{\boldsymbol{U}_{1}=0.035} \frac{\left\|\left(\mathcal{F}_{1}\right)_{H P R O M}-\left(\mathcal{F}_{1}\right)_{H F}\right\|}{\left\|\left(\mathcal{F}_{1}\right)_{H F}\right\|} d \boldsymbol{U}_{1}
$$

As can be observed, the relative errors do not exceed 2.5\%. The Figure also displays the speed-up for each HPROM solution, respect to the HFFEM computational time.

The speedup is estimated by evaluating the computational cost required to solve 10 global steps. The HPROM solutions have been obtained using a single desktop computer i7 $47703.5 \mathrm{GHz}$ and 32Gb RAM. While the full HFFEM solution was obtained in a computer cluster utilizing 96 cores. In this case, the clock time has been 223866.sec.

In Figure 21, we show the iso-maps of the deformation gradient fluctuations that have been obtained with the HFFEM and HPROM solution (using $n_{F}=30$ and $N_{r}=97$ quadrature points). Three components 


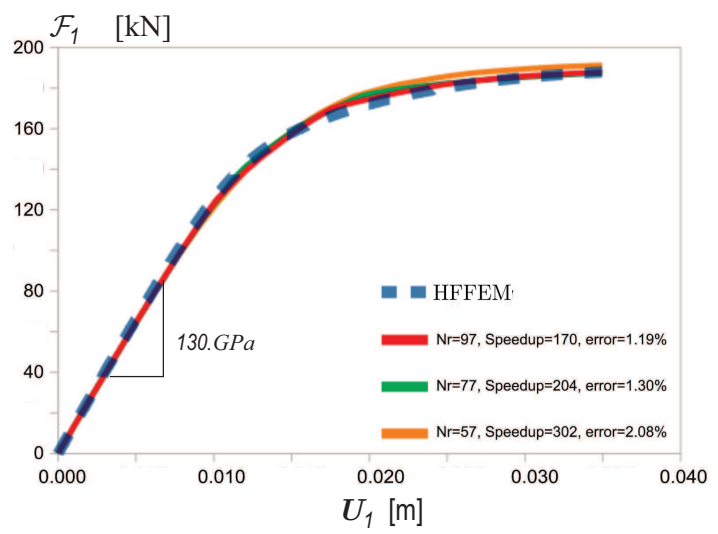

Figure 20: Multiscale simulation of the bar problem. Structural response obtained with the HFFEM and HPROM solutions.

are compared, $\Delta F_{11}, \Delta F_{22}, \Delta F_{12}$ and $\Delta F_{21}$. These solutions correspond to the last simulated load step. In the Figure, it can be seen the strain localization pattern, between voids, which will lead to the macro-stability loss (necking) in subsequent time steps. In the same Figure, observe the distribution of macro-cumulative plastic strains at the same time step.

Figure 22 compares the plots of $\operatorname{det} \boldsymbol{Q}=\operatorname{det}(\boldsymbol{N}(\omega) \cdot \mathbb{A} \cdot \boldsymbol{N}(\omega))^{6}$ for the HFFEM model and HPROM solutions, respectively. The angle $\omega$ at the macro-scale defines the unit normal vector $\boldsymbol{N}$ at the reference configuration for which, the localization tensor is computed. Note that the macro-scale strong-ellipticity indicator is well-captured by the HPROM solution. This is an additional result proving that the effective constitutive tensor $\mathbb{A}$, of the HPROM model, approaches the effective one of the HFFEM solution.

\section{Conclusions}

In this work, the HPROM technique previously presented by the authors in [7], is extended to the case of modeling heterogeneous materials within a multi-scale $F E^{2}$ approach accounting for large elasto-plastic strains (Kouznetsova).

Hyper-reduction is attained by utilizing a specific quadrature scheme based on the ROQ technique applied to the elastic potential energy of the material. The micro-cell volume integrations of the nonlinear terms arising in conventional multiscale material modeling, i.e the micro-scale variational problem, the homogenized stress equations and the homogenized constitutive tensor, are treated with a modified "optimal reduced order quadrature" scheme.

The resulting methodology can be inserted into the general framework of the Lagrangian structure preserving reduction method [9], here generalized to the case of deformable continua in a multiscale modeling context. The essential behind the approach consists of resorting to the fundamental or primitive statement of the problem expressed in terms of a material domain integral. An equivalent statement

${ }^{6}$ The acoustic tensor in indicial notation is $\boldsymbol{Q}_{i k}=\boldsymbol{N}_{j} \mathbb{A}_{i j k l} \boldsymbol{N}_{l}$. 


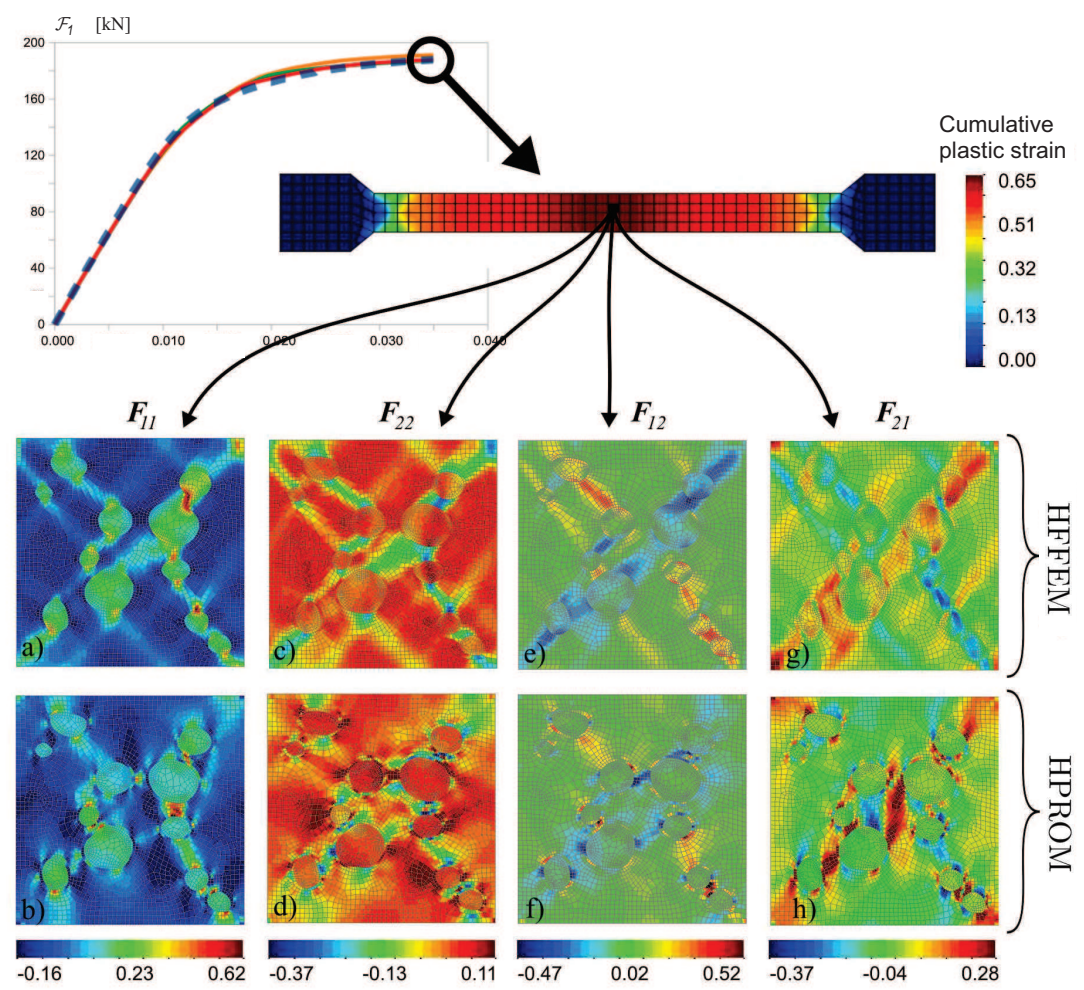

Figure 21: Multiscale simulation of the bar problem. Iso-color maps of deformation gradient fluctuations. Comparison between HFFEM and HPROM solutions.

is obtained by deriving a $R O Q$ in this domain through optimization of the numerical integration rule to minimize the computational cost and maximize the accuracy. Then, the corresponding variational problem is obtained by differentiation with respect to the state variables of that optimized equivalent statement, this constituting the basis for most of the computational cost reduction. This technique, that was previously developed in the previous work by the authors [7] and applied to non-linear multiscale fracture problems undergoing infinitesimal strains [10], is here generalized to the case of non-linear kinematics and non-linear material behavior, and is the main contribution of this work. This proves that the proposed HPROM techniques can be efficiently applied either in non-linear kinematics as well as in non-linear material mechanics under the same general principles.

The numerical assessment has been addressed through a number of examples of increasing complexity (in terms of the number of involved algebraic operations), and the trade-off between HPROM fidelity loss vs. computational speed-ups are remarkable.

Another notable issue is the scalability in terms of speed-ups versus HFFEM complexity. In fact, this scalability is a manifestation that, for a given given micro-structure morphology and specific accuracy (and a sufficient finite element representation of it), the HPROM representations for increasingly refined Finite Element meshes given micro-structure morphology lay on similar linear spaces. In other words, for a given RVE morphology the HPROM computational cost tends to be constant (for a given 

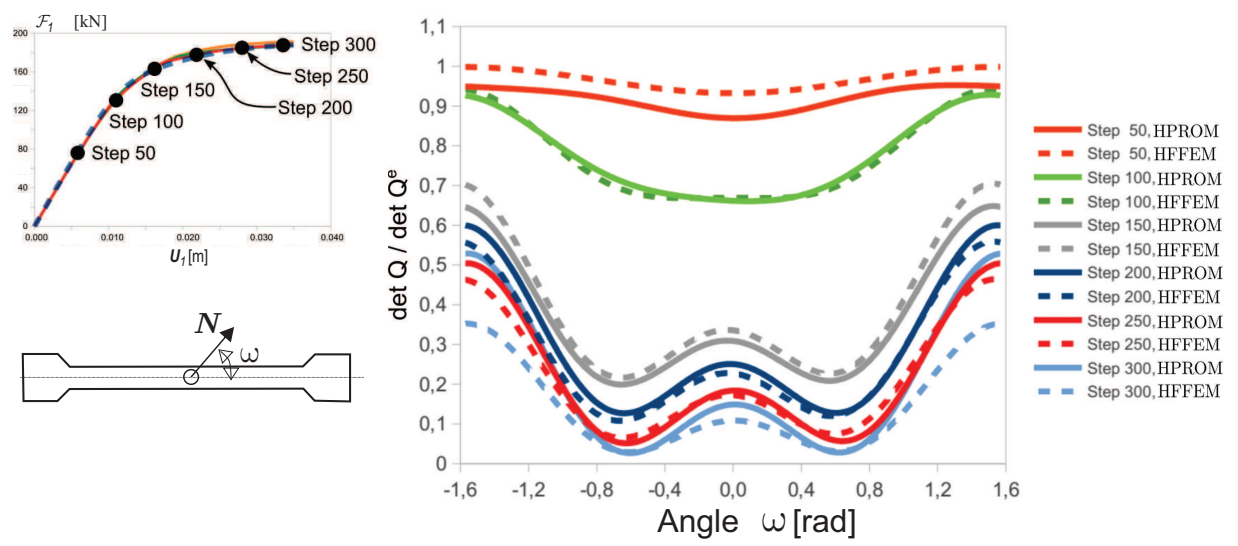

Figure 22: Multiscale simulation of the bar problem. Plots of $\operatorname{det} \boldsymbol{Q}$ computed at the central point of the bar at several load steps.

HPROM complexity $=n_{F} \times N_{r}$ ), and mesh refinement of the HFFEM model does not translate into relevant additional computational times for the resulting HPROM model.

These considerations set new insights, and open a variety of scenarios regarding to industrial applications in material modeling at two length scales, which are currently under study by the authors. They may contribute to mitigate one of the roadblocks influencing the so-called tyranny of scales ([21]) and making material multi-scale modeling techniques evolve toward routinely computational tools.

Finally, although this study has been performed in simplified 2D problems, in the authors' opinion, similar conclusions can be also anticipated valid for 3D cases. This is left to be proven in a future work.

\section{Acknowledgments}

The authors acknowledge the financial support from the European Research Council under the European Unions Seventh Framework Programme (FP/2007-2013) / ERC Grant Agreement N. 320815 (ERC Advanced Grant Project Advanced tools for computational design of engineering materials COMPDES-MAT).

Also, second and fourth authors acknowledge the financial support from CONICET and ANPCyT (grants PIP 2013-2015 631 and PICT 2014-3372).

The authors would like also to acknowledge the support of Dr. Joaquin Hernández, from CIMNE, on the algorithmic and technical aspects of the reduced order integration methods used in this work.

\section{Appendix A. Constitutive model equations}

In this appendix, we summarize the constitutive equations for modeling the micro-cell components of the ferritic ductile iron utilized in Section 5 . The $J_{2}$-plasticity model here adopted is similar to that 
presented in Sections 50-53 of the book [22].

Such as assumed in sub-Section 2.3, we take a multiplicative decomposition of the micro-deformation gradient: $\boldsymbol{F}_{\mu}=\boldsymbol{F}_{\mu}^{e} \boldsymbol{F}_{\mu}^{p}$, where $\boldsymbol{F}_{\mu}^{e}$ and $\boldsymbol{F}_{\mu}^{p}$ are the elastic and plastic deformation gradients, respectively. We also assume an additive decomposition of the free energy, see Eq. (11). In this context, the elastic free energy part is defined by the Henky model given by

$$
\begin{aligned}
\varphi_{\mu}^{e}\left(\boldsymbol{F}_{\mu}, \boldsymbol{F}_{\mu}^{p}\right)= & \frac{1}{2} \lambda\left[\left(\epsilon_{\mu}^{e}\right)_{I}+\left(\epsilon_{\mu}^{e}\right)_{I I}+\left(\epsilon_{\mu}^{e}\right)_{I I I}\right]^{2}+ \\
& +G\left[\left(\epsilon_{\mu}^{e}\right)_{I}^{2}+\left(\epsilon_{\mu}^{e}\right)_{I I}^{2}+\left(\epsilon_{\mu}^{e}\right)_{I I I}^{2}\right],
\end{aligned}
$$

where $\lambda$ and $G$ are the Lamè parameters $\left(\lambda=\frac{E \nu}{(1+\nu)(1-2 \nu))}, G=\frac{E}{2(1+\nu)}\right)$, and $\left(\epsilon_{\mu}^{e}\right)_{i}$ are the logarithmic stretching

$$
\left(\epsilon_{\mu}^{e}\right)_{i}=\log \left(\lambda_{i}^{e}\right) \quad ; \quad \text { for: } i=I, I I, I I I,
$$

being $\lambda_{i}^{e}$ the stretching satisfying

$$
\lambda_{i}^{e}=\text { eigenvalues }\left(\sqrt{\boldsymbol{F}_{\mu}^{e}\left(\boldsymbol{F}_{\mu}^{e}\right)^{T}}\right)
$$

From Eq. (11), the Kirchhoff stress, $\boldsymbol{\tau}$, is given by

$$
\boldsymbol{\tau}_{\mu}=\sum_{i=I}^{i=I I I} \tau_{i}\left(\boldsymbol{n}_{i} \otimes \boldsymbol{n}_{i}\right)
$$

where the principal stresses $\tau_{i}$ (for $\left.i=I, I I, I I I\right)$, are

$$
\tau_{i}=\lambda\left[\left(\epsilon_{\mu}^{e}\right)_{I}+\left(\epsilon_{\mu}^{e}\right)_{I I}+\left(\epsilon_{\mu}^{e}\right)_{I I I}\right]+2 G\left(\epsilon_{\mu}^{e}\right)_{i} \quad \text { for: } i=I, I I, I I I,
$$

and $\boldsymbol{n}_{i}$ are the eigenvectors of the tensor $\left(\sqrt{\boldsymbol{F}_{\mu}^{e}\left(\boldsymbol{F}_{\mu}^{e}\right)^{T}}\right)$. The first Piola-Kirchhoff stress can be computed from $\boldsymbol{P}_{\mu}=\boldsymbol{\tau}_{\mu} \boldsymbol{F}_{\mu}^{-T}$.

The free energy plastic part is defined by

$$
\varphi_{\mu}^{p}\left(\alpha_{\mu}\right)=\frac{1}{2} H_{\mu} \alpha_{\mu}^{2}+\left(\Delta \sigma_{Y}\right)\left(\alpha_{\mu}+\frac{1}{\delta_{\mu}} \exp \left(-\delta_{\mu} \alpha_{\mu}\right)\right)+\sigma_{Y}^{0} \alpha_{\mu} .
$$

with the internal variable $\alpha_{\mu}$ being the cumulative plastic strain which rate is defined by

$$
\dot{\alpha}_{\mu}=\sqrt{\frac{2}{3} \boldsymbol{L}_{p}^{T}: \boldsymbol{L}_{p}} \quad ; \quad \text { with: } \boldsymbol{L}_{p}=\dot{\boldsymbol{F}}_{p} \boldsymbol{F}_{p}^{-1} .
$$

Considering the Eq. (A.6), the radius of the Mises yield function results

$$
\sigma_{Y}\left(\alpha_{\mu}\right)=H_{\mu} \alpha_{\mu}+\left(\Delta \sigma_{Y}\right)\left(1-\exp \left(-\delta_{\mu} \alpha_{\mu}\right)\right)+\sigma_{Y}^{0} .
$$

\section{Conflict of interest}

On behalf of all authors, the corresponding author states that there is no conflict of interest. 


\section{References}

[1] C. Miehe, J. Schotte, J. Schröder, Computational micro-macro transitions and overall moduli in the analysis of polycrystals at large strains, Computational Materials Science 6 (1999) 372-382.

[2] K. Terada, N. Kikuchi, A class of general algorithms for multi-scale analyses of heterogeneous media, Computer methods in applied mechanics and engineering 190 (40) (2001) 5427-5464.

[3] F. Feyel, J. Chaboche, $\mathrm{FE}^{2}$ multiscale approach for modelling the elastoviscoplastic behaviour of long fibre SiC/Ti composite materials, Comput. Meth. App. Mech. Eng. 183 (2000) 309-330.

[4] J. Michel, H. Moulinec, P. Suquet, Effective properties of composite materials with periodic microstructure: a computational approach, Computer methods in applied mechanics and engineering $172(1-4)(1999)$ 109-143.

[5] D. Ryckelynck, Hyper-reduction of mechanical models involving internal variables, International Journal for Numerical Methods in Engineering 77 (1) (2009) 75-89.

[6] J. Hernández, J. Oliver, A. Huespe, M. Caicedo, J. Cante, High-performance model reduction techniques in computational multiscale homogenization, Computer Methods in Applied Mechanics and Engineering 276 (2014) 149-189.

[7] J. Oliver, M. Caicedo, A. Huespe, J. Hernández, E. Roubin, Reduced order modeling strategies for computational multiscale fracture, Computer Methods in Applied Mechanics and Engineering 313 (2017) $560-595$.

[8] C. Farhat, T. Chapman, P. Avery, Structure-preserving, stability, and accuracy properties of the energy-conserving sampling and weighting method for the hyper reduction of nonlinear finite element dynamic models, International Journal for Numerical Methods in Engineering.

[9] K. Carlberg, R. Tuminaro, P. Boggs, Preserving lagrangian structure in nonlinear model reduction with application to structural dynamics, SIAM Journal on Scientific Computing 37 (2) (2015) B153-B184.

[10] J. Oliver, M. Caicedo, E. Roubin, A. Huespe, J. Hernández, Continuum approach to computational multiscale modeling of propagating fracture, Computer Methods in Applied Mechanics and Engineering 294 (2015) 384-427.

[11] P. Sánchez, P. Blanco, A. Huespe, R. Feijóo, Failure-oriented multi-scale variational formulation: micro-structures with nucleation and evolution of softening bands, Computer Methods in Applied Mechanics and Engineering 257 (2013) 221-247.

[12] E. de Souza Neto, R. Feijóo, Variational foundation on multi-scale constitutive models of solids: small and large strain kinematical formulation, LNCC Research \& Development Report No 16.

[13] P. Blanco, P. Sánchez, E. de Souza Neto, R. Feijóo, Variational foundations and generalized unified theory of rve-based multiscale models, Archives of Computational Methods in Engineering 23 (2) (2016) 191-253.

[14] V. Lubarda, D. Benson, On the partitioning of the rate of deformation gradient in phenomenological plasticity, International journal of solids and structures 38 (38) (2001) 6805-6814. 
[15] X. Oliver, C. Agelet, Continuum Mechanics for Engineers. Theory and Problems, UPCommons, URL: http://hdl.handle.net/2117/102979; DOI: 10.13140/RG.2.2.25821.20961, 2017.

[16] J. Hernandez, M. Caicedo, A. Ferrer, Dimensional hyper-reduction of nonlinear finite element models via empirical cubature, Computer methods in applied mechanics and engineering 313 (2017) $687-722$.

[17] A. Yaqub, Ejaz, Rizwan, Me-140 workshop technology, Air University, Department of Mechanical \& Aerospace Engineering.

[18] D. Fernandino, A. Cisilino, S. Toro, P. Sanchez, Multi-scale analysis of the early damage mechanics of ferritized ductile iron, International Journal of Fracture (2017) 1-26.

[19] E. Neto, F. Pires, D. Owen, F-bar-based linear triangles and tetrahedra for finite strain analysis of nearly incompressible solids. part i: formulation and benchmarking, International Journal for Numerical Methods in Engineering 62 (3) (2005) 353-383.

[20] S. Toro, P. Sánchez, A. Huespe, S. Giusti, P. Blanco, R. Feijóo, A two-scale failure model for heterogeneous materials: numerical implementation based on the finite element method, International Journal for Numerical Methods in Engineering 97 (5) (2014) 313-351.

[21] J. Oden, T. Belytschko, J. Fish, T. Hughes, C. Johnson, D. Keyes, A. Laub, L. Petzold, D. Srolovitz, S. Yip, Revolutionizing engineering science through simulation, National Science Foundation Blue Ribbon Panel Report 65, www.nsf.gov/pubs/reports/sbes_final_report.pdf.

[22] J. C. Simo, Numerical analysis and simulation of plasticity, Handbook of numerical analysis 6 (1998) 183-499. 